\title{
Estrogen receptor alpha inhibits mesenchymal and amoeboidal movement of liver cancer cell via $G$ protein subunit alpha 12
}

\author{
Jessica Yun ${ }^{1}$, Mi Jeong Heo ${ }^{1}$, Yun Seok Kim¹, Min Joo Kim², Aree Moon ${ }^{2}$, and Sang Geon \\ $\mathrm{Kim}^{1}$ \\ ${ }^{1}$ Seoul National University \\ ${ }^{2}$ Duksung Women's University
}

November 24, 2020

\begin{abstract}
Background and Purpose Hepatocellular carcinoma (HCC) is the second most common cancer worldwide, demonstrating aggressiveness and mortality more frequently in men than in women. Despite reports regarding the inhibitory ability of estrogen receptor alpha $(\mathrm{ER} \alpha, \mathrm{ESR} 1)$ in certain cancer progression, targets and the basis of underlying gender disparity in HCC worsening remain elusive. Experimental Approach Human HCC samples were used for immunoblottings and immunohistochemistry. Estradiol (E2) was treated to HCC cell lines and were evaluated by immunoblottings, polymerase chain reaction, immunofluorescence, and live imaging. Key Results Here, we report the ability of ER $\alpha$ to transcriptionally inhibit G protein subunit alpha 12 (G $\alpha 12)$. First, using human samples and public database, the expression of ER $\alpha$ and G $\alpha 12$ in HCC was examined. Then, quantitative real-time PCR, chromatin immunoprecipitation-assay, luciferase assay, and immunoblottings confirmed the inhibitory ability of ER $\alpha$ on G $\alpha 12$ and EMT. Additionally, we found microRNA-141 and -200a as downstream targets of the G $\alpha 12$ signaling axis for cancer malignancy regulation under the control of ER $\alpha$. As for in-depth mechanism, PTP4A1 was found to be directly inhibited by microRNA-141 and -200a. G $\alpha 12$ and PTP4A1 promoted epithelial-mesenchymal transition, as well as mesenchymal to amoeboidal transition, antagonized by ER $\alpha$ modulations. Conclusion and Implications The identified targets and ESR1 levels inversely correlated in human specimens, as well as with sex-biased survival rates of HCC patients. Collectively, ER $\alpha$-dependent repression of $\mathrm{G} \alpha 12$ and consequent changes in the G $\alpha 12$ signaling may explain the gender disparity in HCC, providing pharmacological clues for the control of metastatic HCC.
\end{abstract}

Estrogen receptor alpha inhibits mesenchymal and amoeboidal movement of liver cancer cell via G protein subunit alpha 12

Short title: $\mathrm{ER} \alpha$ inhibition of $\mathrm{G} \alpha 12$

Jessica Yun ${ }^{1}$, Mi Jeong $\mathrm{Heo}^{1}$, Yun Seok Kim ${ }^{1}$, Min Joo Kim² ${ }^{2}$ Aree Moon ${ }^{2}$, and Sang Geon Kim ${ }^{3,1, *}, \#$

${ }^{1}$ College of Pharmacy, Seoul National University, Seoul, Republic of Korea, ${ }^{2}$ Duksung Innovative Drug Center, College of Pharmacy, Duksung Women's University, Seoul, Republic of Korea; and ${ }^{3}$ College of Pharmacy and Integrated Research Institute for Drug Development, Dongguk University_Seoul, Goyang 10326, Kyeonggi-do, Republic of Korea

*Correspondence to: SG Kim, Tel: +8231-961-5218; Fax: +8231-961-5206; E-mail: sgkim@dongguk.edu

\#Lead Contact

Word Count : 4303

Acknowledgments: This research was supported by the Bio \& Medical Technology Development Program of the NRF, funded by the Korean government, MSIT (2015M3A9B6074045), and in part by Dongguk 
University fund.

\section{Authors' Contributions}

Study concept and design; acquisition of data; analysis and interpretation of data; drafting of the manuscript; statistical analysis: Jessica Yun and Mi Jeong Heo

Acquisition of data; analysis and interpretation of data; statistical analysis for liver cancer: Jessica Yun, Mi Jeong Heo, and Yun Seok Kim

Acquisition of invasion and migration data and xenograft experiment: Min Joo Kim and Aree Moon

Material and administrative support; analysis and interpretation of data: Jessica Yun, Mi Jeong Heo, and Yun Seok Kim

Study concept and design; analysis and interpretation of data; manuscript writing; critical revision of the manuscript for important intellectual content; obtained funding; administrative, technical, or material support; and study supervision: Sang Geon Kim

\section{Conflict of Interests Statement}

The authors declare no conflicts of interest.

\section{Background and Purpose}

Hepatocellular carcinoma (HCC) is the second most common cancer worldwide, demonstrating aggressiveness and mortality more frequently in men than in women. Despite reports regarding the inhibitory ability of estrogen receptor alpha (ER $\alpha, E S R 1)$ in certain cancer progression, targets and the basis of underlying gender disparity in $\mathrm{HCC}$ worsening remain elusive.

\section{Experimental Approach}

Human HCC samples were used for immunoblottings and immunohistochemistry. Estradiol (E2) was treated to HCC cell lines and were evaluated by immunoblottings, polymerase chain reaction, immunofluorescence, and live imaging.

Key Results

Here, we report the ability of ER $\alpha$ to transcriptionally inhibit G protein subunit alpha 12 (G $\alpha 12)$. First, using human samples and public database, the expression of ER $\alpha$ and G $\alpha 12$ in HCC was examined. Then, quantitative real-time PCR, chromatin immunoprecipitation-assay, luciferase assay, and immunoblottings confirmed the inhibitory ability of ER $\alpha$ on G $\alpha 12$ and EMT. Additionally, we found microRNA-141 and -200a as downstream targets of the G $\alpha 12$ signaling axis for cancer malignancy regulation under the control of ER $\alpha$. As for in-depth mechanism, PTP4A1 was found to be directly inhibited by microRNA-141 and -200a. Ga12 and PTP4A1 promoted epithelial-mesenchymal transition, as well as mesenchymal to amoeboidal transition, antagonized by $\mathrm{ER} \alpha$ modulations.

\section{Conclusion and Implications}

The identified targets and ESR1 levels inversely correlated in human specimens, as well as with sex-biased survival rates of HCC patients. Collectively, ER $\alpha$-dependent repression of G $\alpha 12$ and consequent changes in the Ga12 signaling may explain the gender disparity in HCC, providing pharmacological clues for the control of metastatic HCC.

Key words : liver cancer, metastasis, estrogen receptor alpha, Ga12, PTP4A1

\section{Introduction (516 words)}

Hepatocellular carcinoma (HCC) is the fourth leading cause of death among cancers (Bray et al., 2018). Epidemiologically, HCC has revealed gender disparity, demonstrating higher morbidity and mortality in male patients (Fitzmaurice et al., 2017). The biased favoring females in HCC development and progression 
suggests sex hormones (i.e., estrogens and androgens) may play distinct roles (Barreca et al., 1993). However, the role of estrogens preventing HCC progression, as well as in normal liver functions, remains elusive. In HCC, expression of estrogen receptor alpha $(\mathrm{ER} \alpha)$ appears to inversely correlate with larger tumors, proliferation, migration, and invasion (Naugler et al., 2007; Yang et al., 2012; Liu et al., 2009). In addition, the repression of $\mathrm{ER} \alpha$ causes tumorigenesis in HCC (Bigsby RM and Caperell-Grant A, 2011; Wu et al., 2017). Therefore, it needs to be resolved whether estrogen and ER $\alpha$ signaling mitigate HCC progression, and if so, the underlying basis needs to be elucidated.

Epithelial to mesenchymal transition (EMT) describes the movement of the cytoskeletal organization to allow cells to detach and disseminate by degrading the extracellular membrane (Choi and Diehl, 2009). As HCC demonstrates a poor prognosis owing to its metastatic phenotype, EMT is recognized as a key process in tumor metastasis. Thus, EMT is more frequently observed in metastatic cancer cells (Nieto, 2013; Thiery et al., 2009). Among several biomarkers observed in HCC, G protein subunit alpha 12 (Ga12), gep oncogene, whose gene expression product is overly expressed in metastatic HCC, is a driver gene for HCC progression, mediated by alterations in microRNAs (miRNAs) and mouse double minute 2 homolog expression (Yang et al., 2015; Heo et al., 2014).

Cancer cells adopt different motility for dissemination, presenting an obstacle for developing treatments. Mesenchymal movement forms an elongated phenotype, whereas amoeboid motility presents a round morphology (Nobes and Hall, 1995; Panková et al., 2010). Hence, the invasion/migration of cancer cells consists of not only mesenchymal but also amoeboidal movements (Sahai and Marshall, 2003). Furthermore, it has been recognized that Rho/ROCK may play a role in the amoeboid movement, which is one of the main downstream targets of the G $\alpha 12$ signaling pathway (Seasholtz et al., 1999). Therefore, it is important to examine the ways cancer cells migrate and invade to prevent progression of metastatic cancers.

This study investigated whether ER $\alpha$ level in HCC is associated with that of G $\alpha 12$ as well as the consequent changes observed in overall survival rates of the patients. In cellular models and human sample analyses, we identified the link between ER $\alpha$ and G $\alpha 12$. Molecular studies confirmed the ER $\alpha$ ability to inhibit Ga12 (GNA12), newly revealing miR-141 and -200a as downstream targets of the Ga12 signaling axis, in conjunction with ER $\alpha$ expression change. Moreover, we discovered protein tyrosine phosphatase type IVA 1 (PTP4A1) as a direct target of miR-141 and -200a and the consequent changes in phosphorylated-myosin light chain (p-MLC) levels, a protein responsible for amoeboid movement downstream of G $\alpha 12$ pathway. Functionally, we demonstrated the regulatory effect of $\mathrm{G} \alpha 12$ on amoeboid motility under the inhibitory control of ER $\alpha$, clarifying the molecules responsible for HCC malignancy, as well as for gender disparity and prognosis in HCC patients.

\section{Materials and Methods (1,921)}

\section{Antibodies and reagents}

ICI 182,780 (I4409) and anti- $\beta$-actin (A5441) antibodies were purchased from Sigma-Aldrich (St Louis, MO, USA). Doxycycline (324385) and estrogen (3301) were obtained from Sigma-Aldrich (St Louis, MO, USA). Anti-ER $\alpha$ (8002), anti-Vimentin (32322), anti-ZEB1 (25388), and anti-G 12 (515445) antibodies were provided from Santa Cruz Biotechnology (Santa Cruz, CA, USA). Antibodies directed against p-MLC (ser19) were obtained from Cell Signaling (Beverly, MA, USA) (3671). Anti-E-cadherin antibody was purchased from BD Biosciences (San Jose, CA, USA) (610181). Anti-PTP4A1 antibody was supplied from Proteintech (Chicago, IL, USA) (11508-1-AP). Antibodies obtained from Santa Cruz Biotechnology were used at a ratio of 1:1000 dilution, whereas the others were done in 1:5,000-10,000 dilution.

\section{Cell culture}

HepG2 cell (RRID: CVCL_0027) was supplied from ATCC (Manassas, VA). HEK293A and HEK293T cells were provided from American Type Culture Collection (Manassas, VA, USA). Huh7, SK-Hep1, SNU387, and SNU449 cell lines were purchased from Korean Cell Line Bank (KCLB, Seoul, Korea). All human cell lines had been authenticated using STR profiling within the last three years. All experiments were 
performed with mycoplasma-free cells. Huh7, HepG2, HEK293A, HEK293T, SNU449, and SK-Hep1 cells were maintained in Dulbecco's modified Eagle's medium containing $10 \%$ fetal bovine serum, 50 units $/ \mathrm{mL}$ penicillin, and $50 \mu \mathrm{g} / \mathrm{mL}$ streptomycin at $37^{\circ} \mathrm{C}$ in a humidified atmosphere containing $5 \% \mathrm{CO} 2$. SNU387 cells were cultured in RPMI 1640 supplemented with $10 \%$ fetal bovine serum and $1 \%$ penicillin-streptomycin at $37^{\circ} \mathrm{C}$ in a humidified atmosphere containing $5 \% \mathrm{CO} 2$.

\section{Immunohistochemistry}

Formalin-fixed, paraffin-embedded HCC and NT liver samples were cut using a microtome into $4 \mu \mathrm{m}$ thickness. The tissue sections were mounted on glass slides. Antibodies of interest (ER $\alpha, \mathrm{G} \alpha 12$, PTP4A1, p-MLC, and vimentin) were incubated overnight at room temperature with the slide-mounted method.

\section{Human liver samples}

The 59 paired samples of HCC and NT tissue were obtained from 187 patients who underwent curative surgical resection and donated samples to the Bio-Resource Center at the Asan Medical Center (Seoul, South Korea) between the years of 2006 and 2009. Tumors were classified according to the World Health Organization pathologic classification system. All patients provided informed consents, and the study protocol was approved by institutional review board of Asan Medical Center (no. 2012-0133) in accordance with the ethical guidelines of the 1975 Declaration of Helsinki.

\section{Transient transfection}

ER $\alpha$ and PTP4A1 expression clones were purchased from Genecopoeia (Rockville, MD). The empty plasmid, pcDNA3.1 and pCMV, was used for mock transfection. Cells were transfected with the indicated plasmid $(1 \mu \mathrm{g})$ using Lipofectamine 2000 (Invitrogen, Carlsbad, CA) according to the manufacturer's instructions. Scrambled small interfering RNA (control siRNA), or siRNA specifically directed against ER $\alpha, \mathrm{G} \alpha 12$, and PTP4A1 were purchased from Dharamacon (Lafayette, CO, USA). The cells were transfected with plasmid or small-interfering RNA (siRNA) using Lipofectamine 2000 transfection reagent (Invitrogen, Carlsbad, CA) for $72 \mathrm{~h}$.

\section{Establishment of stable cell lines}

The virus productions were carried out using HEK293T cells. Briefly, cells were transfected with lentiviral DNA constructs combined with the lentiviral packaging plasmids pMD2G, pMDLg and pRSV-Rev

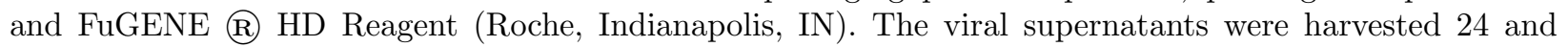
48 hours post-transfection and used for infections. Then $300 \mu \mathrm{L}$ of virus containing culture medium was added to the cells. To establish stable cell lines, SK-Hep1 cells were infected with rtTA3 lentivirus and selected with hygromycin. The selected cells were infected with tetracycline (Tet)- On ER $\alpha$ lentiviruses and were selected with puromycin. A Tet On lentiviral plasmid containing the ER $\alpha$ sequence was synthesized by Cosmogenetech (Seoul, Korea). The plasmid TetO-FUW-pgk-puro from Emily Dykhuizen (Addgene plasmid \# 85747), pLenti CMV rtTA3 Hygro (w785-1) from Eric Campeau (Addgene plasmid \# 26730), $\mathrm{pMDLg} / \mathrm{pRRE}$ from Didier Trono (Addgene plasmid \# 12251), pRSV-Rev from Didier Trono (Addgene plasmid \# 12253), and pMD2.G from Didier Trono (Addgene plasmid \# 12259) were provided by Addgene (http://www.addgene.org). For conditional ER $\alpha$ overexpression, doxycycline (Dox) $2 \mu \mathrm{g} / \mathrm{ml}$ was treated in SK-Hep1 cells.

\section{Bioinformatics analyses}

RNA-seq data from Gene Expression Omnibus (GEO, https://www.ncbi.nlm.nih.gov/geo/) (GSE40367, GSE94016) was subjected to Kyoto Encyclopedia of Genes and Genomes (KEGG) enriched pathway analysis (pathway in cancer) using the DAVID 6.7 (http://david.avcc.ncifcrf.gov/ref) online bioinformatics tool. Gene interaction analysis between the clustered genes was achieved according to STRING v9.1 database (http://string-db.org). The potential targets of miRNAs of interest were extracted from TargetScan database (http://targetscan.org). Gene ontology-based functional groups of miRNA targets were searched using the DAVID 6.7 (http://david.avcc.ncifcrf.gov/ref) online bioinformatics tool. 
Transcriptome data GEO (https://www.ncbi.nlm.nih.gov/geo/) (GSE9843, 40367, 76427) was analyzed using gene set enrichment analysis (GSEA) 3.0 software. 'Biological process of gene ontology (GO)' from Molecular Signature Database (MSigDB, http://software.broadinstitute.org/gsea/msigdb) v6.2 was employed. False discovery rate (FDR) was used for statistical significance assessment of normalized enrichment score (NES). The criterion for statistical significance was set at $\mathrm{FDR}<0.25$. Heat map represents the respective leading-edge subsets of the most upregulated genes.

\section{Luciferase activity}

The plasmids of Luc-GNA12 -3'untranslated region (UTR) and Luc-PTP4A1 -3'UTR were specifically synthesized (GeneCopoeia, Rockville, MD), and were used in luciferase reporter assays. The plasmid contains firefly luciferase gene fused to the 3'UTR of human G $\alpha 12$ (or PTP4A1), and Renilla luciferase that functions as a tracking gene. Luciferase activity assays were done according to manufacturer protocols. Briefly, HEK293A cells were seeded in 6-well plates, and co-transfected with ER $\alpha$ and wild type (WT) vectors, and Ga12 3' UTR WT reporter. Similarly, SNU449 or HepG2 cells were seeded in 6-well plates, and cotransfected with miR-141 and -200a mimic (or antisense oligonucleotide (ASO)), and PTP4A1 3'UTR WT reporter. The cells were harvested following transfection for $48 \mathrm{~h}$. Firefly and Renilla luciferase activities were measured sequentially with the dual luciferase assay kit (GeneCopoeia). The activities were normalized with Renilla luciferase activities and expressed in relative luciferase activity units. The cells were transfected with plasmid using Lipofectamine 2000 transfection reagent (Invitrogen, Carlsbad, CA) for $48 \mathrm{~h}$.

\section{Chromatin immunoprecipitation (ChIP) assays}

ChIP assays were carried out using the EZ ChIP Kit (Millipore, Billerica, MA, USA) according to the manufacturer's protocol. SK-Hep1 cells were treated with Dox and E2 for $24 \mathrm{~h}$ each, and then formaldehyde was added to the cells to a final concentration of $1 \%$ for cross-linking of chromatin. The ChIP assay was performed according to the ChIP assay kit protocol (Upstate Biotechnology, Lake Placid, NY). PCR was done using the primers flanking the putative ER $\alpha$ binding sites located in the promoter region of human GNA12 gene. IgG immunoprecipitation represents negative control. The primer sequences used for the qPCR analysis were as follows:

Site \#1

\section{Forward: 5'- TCAAGTGATCCTCCCACCTC -3'}

Reverse: 5'- TGGCTTCATTTCTGGGTTCT -3'

Site \#2

Forward: 5'- CAAGGTTGTCAGGGAGTTGG -3'

Reverse: 5'- GGCACGGTGACTCACAACTA -3'

\section{Immunocytochemistry}

SK-Hep1 cells treated with Dox and E2 $(24 \mathrm{~h})$ were grown on a coverslip and were fixed in a $4 \%$ paraformaldehyde solution followed by permeabilization with $0.3 \%$ Triton X-100 (Sigma Aldrich). After washing with PBS, the cell samples were immunostained with antibodies directed against ER $\alpha$ and G $\alpha 12$. Alexa Fluor 488 goat anti-mouse IgG (Invitrogen) and Alexa Fluor 594 goat anti-rabbit IgG (Invitrogen) were used as secondary antibody. After incubation, 1/10 diluted drop of Hoechst was treated for another 15 minutes. Then, the samples were cover-slipped with mounting media, and were examined using laser-scanning confocal microscopes (Leica TCS NT and Leica TCS8, Leica Microsystems, Wetzlar, Germany).

$R N A$ isolation and quantitative reverse transcription-polymerase chain reaction ( $P T$ - $P C R$ ) assays Total RNAs were extracted with the Trizol (Invitrogen, Carlsbad, CA, USA). qRT-PCR assays for messenger RNAs (mRNAs) were performed using StepOne real-time PCR instrument (Thermo Fisher Scientific) and SYBR Premix Ex TaqII kit (Takara Bio, Shiga, Japan), whereas those for miRNAs were carried out using 
miScript SYBR Green PCR Kit (Qiagen) according to the manufacturer's instruction. The primer sequences were as follows:

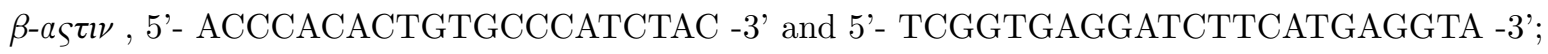
18s RNA , 5'- GTAACCCGTTGAACCCCATT -3' and 5'- CCATCCAATCGGTAGTAGCG -3'; GNA12 , 5'- CTCAAGGGCTCAAGGGTTCTT -3' and 5'- CAGGAACATCCCATGCTTCTC -3'; ESR1 , 5'- GAGTAACAAAGGCATGGAGCA -3' and '5- ATGAAGTAGAGCCCGCAGTG -3'; PRL-1 , 5'- ACCAATGCGACCTTAAACAAA -3' and 5'-AATCTGGTTGGATGGTGGTG-3'; and VIM , 5'- AAGGTCAAGACGTGCCAGAG -3' and 5'- TTCCAGTTCTGCACGGTCTC -3' qRT-PCR assays for miRNAs

cDNA was generated from equal amounts of total RNA per sample $(1 \mu \mathrm{g})$ using the miScript Reverse Transcription kit (Qiagen GmbH, Hilden, Germany). The reaction mixture containing reverse transcription product, $2 \times$ QuantiTect SYBR Green PCR Master Mix, $10 \times$ miScript Universal Primer, and primers was incubated at $95^{\circ} \mathrm{C}$ for $15 \mathrm{~min}$, followed by 40 amplification cycles of $94^{\circ} \mathrm{C}$ for $10 \mathrm{~s}, 55^{\circ} \mathrm{C}$ for $30 \mathrm{~s}$, and $70^{\circ} \mathrm{C}$ for $30 \mathrm{~s}$. The threshold cycle $(\mathrm{Ct})$ was defined as the fractional cycle number at which the fluorescence passed the fixed threshold. Transcripts of U6 small RNA were quantified using the Hs_RNU6B_2 miScript Primer Assay (Qiagen, Hilden, Germany) for normalization of miRNA levels. The relative expression values were normalized to the internal control using $2^{-\mathrm{Ct}}$. The following primer sequences were used:

Human-miR-141-3p, 5'-TAACACTGTCTGGTAAAGATGG-3'

Human-miR-200a-3p, 5'-TAACACTGTCTGGTAACGATGT-3';

Western blotting assays

Whole-cell lysates were prepared according to the established procedures. Proteins were resolved by sodium dodecyl sulfateGmbH, Hrylamide gel electrophoresis, immunoblotted with the antibody of interest, and were visualized using an ECL chemiluminescence detection kit (Amersham Biosciences, Amersham, UK). At least three independent experiments were carried out with separate samples.

Cell culture in 3D ECM gels and time-lapse imaging

Three-dimensional (3D) collagen type I gel model was prepared using rat tail collagen type I (Corning). Collagen I mixtures $(2.5 \mathrm{mg} / \mathrm{ml})$ were prepared by adding the appropriate volumes of $10 \times$ reconstitution buffer (260 mM sodium bicarbonate and $200 \mathrm{mM}$ HEPES) and 10× RPMI (Sigma Aldrich). To adjust the $\mathrm{pH}$ of the collagen solution to 7.2-7.4, an ice-cold solution of $2 \mathrm{~N} \mathrm{NaOH}$ was used. The neutralized collagen solution was incubated on ice for 3-5 min to allow the $\mathrm{pH}$ to equilibrate, and then the solution was centrifuged at $10,000 \mathrm{~g}$ for $3 \mathrm{~min}$ at $4^{\circ} \mathrm{C}$ to eliminate air bubbles. Time-lapse DIC cell images were captured with an IX81-ZDC (Olympus, Japan) for $24 \mathrm{~h}$ at $37^{\circ} \mathrm{C}$. The microscope was equipped with a Chamlide Incubator System (Live Cell Instrument, Korea), and the environmental chamber mounted on the microscope was constantly maintained at $37^{\circ} \mathrm{C}, 5 \% \mathrm{CO} 2$, and $95 \%$ humidity.

\section{$3 D$ immunofluorescence analysis}

Cells were cultured within polydimethylsiloxane prepolymer (PDMS) glass coverslips (Song et al., 2017), and fixed directly with $4 \%$ formaldehyde for $30 \mathrm{~min}$ at room temperature and subsequently treated with 100 $\mathrm{mM}$ glycine to quench any residual aldehyde groups. After washing with PBS, the cells were permeabilized with $0.5 \%$ Triton X-100 for 30 min at RT and blocked for $2 \mathrm{~h}$ with PBS containing $3 \%$ BSA. In some cases, the cells were stained with either fluorescein-labeled phalloidin (Molecular Probes, 1:250) or Alexa Fluor@ 488-labeled anti-cortactin (Millipore, 1:200) at $4^{\circ} \mathrm{C}$ overnight. The cells were then washed with wash buffer (130 mM NaCl, $13 \mathrm{mM} \mathrm{Na2HPO4,} \mathrm{and} 3.5 \mathrm{mM} \mathrm{NaH2PO4,} \mathrm{pH} \mathrm{7.4).} \mathrm{The} \mathrm{nuclei} \mathrm{were} \mathrm{counterstained} \mathrm{with}$ Hoechst. Images were captured at $37^{\circ} \mathrm{C}$ using a confocal microscope with a Nikon Plan Apochromat $40 \times$ or 
$60 \times / 1.4$ N.A. oil objective (Nikon Eclipse Ti, Nikon, Japan) and analyzed using the NIS software (Nikon) or IMARIS imaging software (Bitplane AG, Zurich, Swiss). The confocal z-stack images obtained from NIS software were reconstructed into 3D images with the aid of the IMARIS software, Easy 3D mode. The visualization analysis of select images was performed using the ImarisColoc and Surpass module.

\section{Statistical analysis}

Statistically significant differences were assessed by the Student's t-test. The data were expressed as the mean \pm SEM. Coefficients of correlation (r) were determined by the Pearson's correlation method. The Kaplan-Meier method was used for survival analysis. Statistical calculations of Pearson's correlation and Kaplan-Meier method were done using SPSS 20.0 and R.

\section{Data availability}

The data that support the findings of this study are available from the corresponding author upon reasonable request. All GEO data were from NCBI Gene Expression Omnibus (GEO accession number: GSE54236, GSE76427, GSE40367, GSE9843, and GSE94016).

\section{Results (2,186 words)}

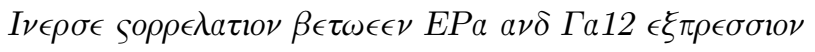

To investigate the relationship between the progression of HCC and ER $\alpha$, we first analyzed a Gene Expression Omnibus (GEO) dataset including various stages of HCC tumors (GSE40637) and generated a heat map. Among the top five genes mostly upregulated as HCC progresses, we focused on G $\alpha 12$ as a potential molecule affected by ER $\alpha$ (Fig. 1A ). The result obtained from the STRING analysis showed the predicted interaction between ESR1 and the genes from Fig. 1A that had significant changes as HCC progresses. Nonetheless, any direct interaction between $\mathrm{ER} \alpha$ and $\mathrm{G} \alpha 12$ is yet unknown (Fig. 1B ). Given the impact of increased G $\alpha 12$ on the EMT and progression of HCC and elusive relationship between ER $\alpha$ and G $\alpha 12$, we wondered whether sex difference affects GPCR activity. In Gene Set Enrichment Analysis (GSEA) using HCC human data (GSE 76427), gene sets associated with EMT and GPCR ligand binding showed significant enrichment in male patients with HCC (Fig. 1C ). In particular, gene set termed as 'EMT' was the only significantly $(\mathrm{FDR}<0.25)$ enriched gene sets in 'HALLMARK' (Supplementary 1). Indeed, the relationship between $\mathrm{ER} \alpha$ and G $\alpha 12$ may be the solution to understand the worsening of HCC, especially in males.

To extend this finding, we employed the GEO dataset in Fig. 1Aand used Pearson correlation analysis to detect transcript levels. As expected, a strong negative relationship was revealed between GNA12 and ESR1, but not between GNA12 andESR2 or G protein-coupled estrogen receptor 1 (GPER1)(Fig. 1D , upper). Moreover, we confirmed that only ESR1 was significantly decreased in metastatic HCCs (Fig. 1D, lower). On this basis, we analyzed the GNA12 and ESR1 from another GEO dataset and observed that GNA12 mRNA levels were lower in patients presenting high levels of ESR1 mRNA (Fig. 1E ).

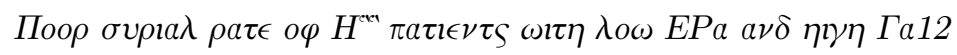

After identifying the effect of ER $\alpha$ on HCC EMT via inverse relationship between G $\alpha 12$, we then analyzed the effect of ER $\alpha$ on the survival rate of HCC patients (Supplementary Table 1), and found that a group of patients with low ER $\alpha$ and high G $\alpha 12$ expression showed the poorest overall or recurrence-free survival rates; however, a group of high $\mathrm{ER} \alpha$ and low G $\alpha 12$ expression demonstrated the opposite effect. The cutoff for strong $\mathrm{G} \alpha 12$ intensity was set at ' $>3$-fold' difference in $\mathrm{HCC} / \mathrm{NT}_{\text {avg }}$ (Fig. $2 \mathbf{A}$, left and middle). Consistently, analysis of GEO dataset also showed the lowest overall survival rate with high G $\alpha 12$ and low ER $\alpha$, whereas those with low G $\alpha 12$ and high ER $\alpha$ had the best outcomes (Fig. 2A , right). Next, we examined the expression of vimentin in the same samples and observed a significant negative association with ESR1, while detecting a positive correlation with GNA12 (Fig. 2B ).

Notably, we examined the ER $\alpha$ expression in the tumor section of tissues from HCC patients and found that $\mathrm{ER} \alpha$ is lowly expressed while G $\alpha 12$ is highly expressed compared with non-tumor section (Fig. 2C ). Moreover, we used immunohistochemistry staining to compare ER $\alpha$ and G $\alpha 12$ levels; ER $\alpha$ expression was 
lower in tumorous tissues compared with non-tumorous tissues, consistent with an inverse relationship of high G $\alpha 12$ expression in tumorous tissues (Fig. 2D ). These results support that G $\alpha 12$ expression is negatively correlated with $\mathrm{ER} \alpha$, suggesting patients with higher ER $\alpha$ have higher overall survival and recurrence-free survival rates, proposing a possible protective role of ER $\alpha$ in HCC progression.

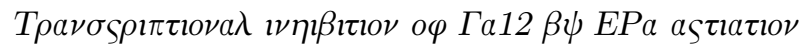

To elucidate the underlying effect of ER $\alpha$ on HCC progression via G $\alpha 12$, we incorporated a 'Tet-On system' to establish a stable cell line that selectively expresses ER $\alpha$ (Fig. 3A , left). As expected, treatment of Tet O-ER $\alpha$ cells with doxycycline (Dox) decreased G $\alpha 12$ mRNA levels (Fig. 3A , right and Supplementary 2A ). Since the classical mechanism of ER $\alpha$ action involves estrogen binding to receptors in the cell and the activated ER $\alpha$ binds to specific response elements known as estrogen response elements (EREs) located in target genes. To corroborate the role of ER $\alpha$ in regulating G $\alpha 12$ transcription, qRT-PCR was performed to assess the effect of modulating ER $\alpha$ expression at the level of G $\alpha 12$ mRNA. Using an ER $\alpha$ overexpression vector, we demonstrated that overexpression of ER $\alpha$ induced the downregulation of G $\alpha 12$ in SNU449 cells (Fig. 3B , left and Supplementary 2B ). Silencing endogenous ER $\alpha$ by using siRNAs led to an upregulation of Ga12 in HepG2 cells (Fig. 3B right and Supplementary 2B ). Furthermore, in SNU387 cells treated with E2 time-dependently, the inhibitory effect was found since GNA12mRNA levels were significantly decreased (Fig. 3C).

To validate the inhibitory effect of ER $\alpha$ on GNA12, we sought an ERE binding site within the GNA12 promoter. Using the ChIP assay, we observed that ER $\alpha$ is a novel inhibitory regulator of G $\alpha 12$ (Fig. 3D ). The luciferase activity assay confirmed that $\mathrm{ER} \alpha$ binds to the Ga12 promoter region. A wildtype GNA12 promoter-luciferase vector was transfected in HEK293A cells and ectopic overexpression of ER $\alpha$ significantly decreased the GNA12 promoter activity (Fig. 3E ). Lastly, using an immunocytochemistry assay, we found that Dox and E2 treatment significantly inhibited Ga12 expression in HCC cells (Fig. 3F ). These results indicate that $\mathrm{ER} \alpha$ directly inhibits the transcription of $\mathrm{G} \alpha 12$.

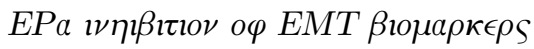

To consolidate the role of ER $\alpha$ in suppressing G $\alpha 12$ and EMT, immunoblottings were done to demonstrate the suppression of G $\alpha 12$ and EMT related proteins (vimentin, zinc finger E-box-binding homeobox 1 (ZEB1), and epithelial cadherin (E-cadherin)) (Fig. 4A ). Furthermore, we confirmed the inhibitory effect of ER $\alpha$

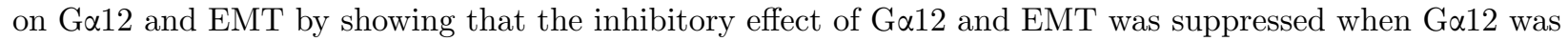
overexpressed (Fig. 4B ). Additionally, the expression levels of ER $\alpha$ and G $\alpha 12 /$ EMT marker proteins were examined using transient and stable ER $\alpha$ transfection (Fig. 4C ). Inversely, ER $\alpha$ silencing increased the levels of Ga12 and EMT markers (Fig. 4D ).

We further demonstrated the suppression of G $\alpha 12$ and other EMT markers in the female-derived cell line SNU387 time-dependently (Fig. 4E ). Consistently, treatment with fulvestrant (ICI 182,780), an antagonist of E2 and ER $\alpha$ down-regulator, promoted the expression of G $\alpha 12$ and EMT markers (Fig. $4 \mathbf{F}$ ). We corroborated the association between ER $\alpha$ and EMT by measuring their endogenous protein levels in several cell lines; mesenchymal-typed HCC cells (SK-Hep1 and SNU449) exhibited lower levels of ER $\alpha$ when compared with epithelial-typed cells (Huh7 and HepG2). Also, SNU387 was used as a positive control as it is a female mesenchymal-typed HCC cell (Fig. 4G ). Our results show that ER $\alpha$ suppression of EMT results from G $\alpha 12$ inhibition.

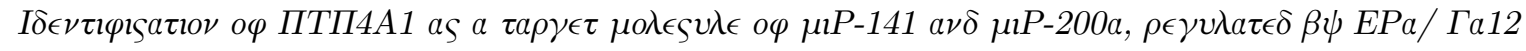

To dissect the mechanism of how the $\mathrm{ER} \alpha / \mathrm{G} \alpha 12$ axis can alter HCC progression, we focused on miRNAs as potential downstream molecules. First, our laboratory previously has performed microarray analyses to assess alterations in miRNA expression in Ga12QL-Huh7 cells and found downregulated and upregulated miRNAs by G $\alpha 12$ (Yang et al., 2015). Here, G $\alpha 12 \mathrm{QL}$ indicates a consistently active mutant form of G $\alpha 12$. Then, common miRNAs were sorted from the list of miRNAs upregulated by estrogen (Huang et al., 2015; Cohen and Smith, 2014) and downregulated by G $\alpha 12$ overexpression. Surprisingly, only two miRNAs were controlled 
by the ER $\alpha$-G $\alpha 12$ axis: miR-141 and -122 (Fig. 5A ). We selected miR-141 for further investigations as miR122 did not express gender disparity while miR-141 did in our human samples (Supplementary 3A and Fig.7C ). We then found that miR-200a presented the same seed sequences as miR-141 (Kehl et al., 2017).

To investigate whether the screened miRNAs are undoubtedly regulated by $\mathrm{ER} \alpha-\mathrm{G} \alpha 12$, we used the HCC cell line stably transfected with an ER $\alpha$ overexpression vector and validated the upregulation of miR-141 and -200 a (Fig. 5B , left). In contrast, ER $\alpha$ was silenced using siRNA, downregulating miR-141 and -200a in HepG2 cells (Fig. 5B , right). Similar results were obtained using another cell line; SNU387 treated with E2 indicated increased level of miR-141 and -200a (Fig. 5C ).

In Fig. 5D , we found that when Ga12 is silenced (Supplementary 4A ), miR-141 and -200a levels increase. Finally, we used Tet-O-ER $\alpha$ cells to transfect the G $\alpha 12$ overexpression vector and simultaneously modulated $\mathrm{ER} \alpha$ and $\mathrm{G} \alpha 12$. The reinforced expression of G $\alpha 12$ prevented $\mathrm{ER} \alpha$ overexpression from increasing miR-141 and -200 a (Fig. 5E ). Hence, ER $\alpha$ may regulate miR-141 and -200a through suppression of Ga12.

Having identified the effect of ER $\alpha-G \alpha 12$ on miR-141 and -200a, we investigated the target of miR-141 and $-200 \mathrm{a}$, a protein possibly implicated in the aggressiveness of HCC. We used Targetscan, a bioinformatics analysis tool, to select the putative targets regulated by miR-141 and -200a. Among the 500 putative targets generated, we did cluster analysis using gene ontology and selected the pathway that regulates cell migration (Supplementary 4B ). Protein tyrosine phosphatase 4A1 (PTP4A1) was chosen for further analysis since phosphatases are emerging therapeutic targets and the role of PTP4A1 in migration and invasion is well known but the regulator is yet elusive (Fig. 5F ). Then, we found a putative binding site of miR-141 and -200a at the 3'UTR of PTP4A1 mRNA (Fig. 5G , left). We next used the luciferase assay to confirm the binding of miR-141 and 200a and PTP4A1 3'UTR region (Fig. 5G , left). As miRNAs may degrade target proteins (Bartel, 2004), we investigated the possibility of PTP4A1 mRNA regulation, which failed to show significant changes due to ER $\alpha$ modulation (Supplementary 4D ). To confirm the role of miR-141 and -200a in regulating PTP4A1 in the $\mathrm{ER} \alpha / \mathrm{G} \alpha 12$ axis, in vitro assays were done after enhancing or silencing the miRNA and $\mathrm{ER} \alpha / \mathrm{G} \alpha 12$. Transfection with miR-141 and -200a mimic or antisense oligonucleotide (ASO) did not influence PTP4A1 mRNA (Supplementary 4C ). Furthermore, miR-141 and -200a mimic decreased PTP4A1 protein levels while ASO increased PTP4A1 protein levels (Fig. 5G, right). Consistently, ER $\alpha$ overexpression decreased PTP4A1, whereas ER $\alpha$ silencing increased PTP4A1 (Fig. 5H ). Moreover, the effect of ER $\alpha$ and $\mathrm{G} \alpha 12$ on PTP4A1 was confirmed through western blotting (Fig. 5I ). In addition, modulation of PTP4A1 on HCC cells affected EMT markers (Fig. 5J ). These results demonstrated that miR-141 and -200a directly inhibits PTP4A1 translation by targeting the 3' UTR region.

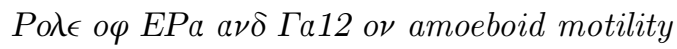

When cancer cells migrate, they have two types of mode: mesenchymal and amoeboid (Sahai and Marshall, 2003), as shown in Fig. 6A . The amoeboid features include membrane blebbing, cell rounding, and increased invasion (Poincloux et al., 2011; Liu et al., 2015; Crosas-Molist et al., 2017). After identifying the link between miR-141/-200a and PTP4A1, as well as the effects of PTP4A1 on its role in HCC progression, we attempted to find whether ER $\alpha$ regulates mesenchymal-amoeboid transition (MAT). Through the analysis of GSEA (GSE9843), we were able to discover many MAT-related pathways increased in male HCC patients (Fig. 6B , Supplementary 5).

Then, we analyzed the morphology and blebbing of SK-Hep1 cell population, presenting both control and $\mathrm{ER} \alpha$ overexpression, in the 3D matrix using live-cell imaging technique (Fig. $6 \mathrm{C}$ ). In the 3D environment, the migration of invasive SK-Hep1 cells showed less blebbing following the overexpression of ER $\alpha$. To confirm the effect of $\mathrm{ER} \alpha$ on amoeboid-blebbing behavior, we examined the levels of the active form of myosin light chain (Ser19) (p-MLC), an amoeboid gold standard marker, in SK-Hep1 cells modulating ER $\alpha$. The confocal assay showed increased expression of p-MLC, as well as its rear distribution (Fig. 6D ), promoting amoeboid movement in the vehicle group (Wilkinson et al., 2005). Next, we examined the protein level of p-MLC by controlling ER $\alpha$ expression. Bona fide, ER $\alpha$ overexpression suppressed p-MLC, whereas siER $\alpha$ increased p-MLC (Fig. 6E ). Moreover, the overexpression of G $\alpha 12$ prevented ER $\alpha$ from inhibiting p-MLC levels 
(Fig. 6F ). Thus, ER $\alpha$ negatively regulates MLC phosphorylation and thereby actomyosin contractility and increased cell migration. Since RhoA/ROCK is a well-established molecule that phosphorylates MLC, and is a downstream molecule of G $\alpha 12$ (Hart et al., 1998), we postulated that p-MLC may be a functional molecule under the control of $\mathrm{ER} \alpha / \mathrm{G} \alpha 12$ axis.

Changes in PTP 4A1, $p$-MLC, and miRNAs in xenograft animal dataset and human samples

In the analysis of publicly available GEO dataset (Yang et al., 2018), the ESR1 level decreased under the situation of metastasis, whereas GNA12 and PTP4A1 increased. We confirmed the negative relationships between ESR1 and GNA12 (orPTP4A1) using Pearson correlation analysis (Fig. 7A ).

To further explore the relationship between $\mathrm{ER} \alpha / \mathrm{G} \alpha 12$ and the target molecules, we did immunoblottings for PTP4A1 and p-MLC. Respectively, samples of tumorous regions showed significantly elevated PTP4A1 and p-MLC levels (Fig. 7B ). Additionally, we observed the histological expression of these molecules in HCC patients, revealing similar results. PTP4A1 and p-MLC expression showed an inverse relationship with $\mathrm{ER} \alpha$ while showed a positive relationship with metastatic proteins (Vimentin and G $\alpha 12$ ) (Fig. 7C ).

To associate the above in vitro results with human HCC progression and further explain the gender disparity, we did qRT-PCR assays for miR-141 and -200a. These miRNAs show positive correlations with ESR1 mRNA levels in HCC patients (Fig. 7D , left). Also, miR-141 and -200a were significantly elevated in female patients (Fig. 7D , right). Furthermore, males demonstrated higher Ga12, PTP4A1 and p-MLC levels when compared with those observed in females (Fig. 7E ). As the expression of ER $\alpha$ in males was one-fifth of that expressed in females (Cooke et al., 2017), the suppression of G $\alpha 12$ would be inhibited. Consequently, miR-141 and -200a were downregulated, and inversely PTP4A1 was increased. In addition, p-MLC was activated as G $\alpha 12$ activates RhoA/ROCK/MLC (Fig. 7F ).

\section{Discussion (1,355 words)}

The incidence of HCC varies worldwide; however, in almost all populations, the male to female ratio averages between 2:1 and 4:1 (Fitzmaurice et al., 2017). HCC is a complex heterogeneous tumor with many genomic alterations (Cancer Genome Atlas Research Network, 2017). To overcome this complexity, an effective combination of molecular therapies is crucial. In addition, the sex bias and consequent changes in major biomarkers suggest the need for personalized therapy (Natri et al., 2019). There is a report indicating that estrogen ameliorates HCC through inhibition of IL-6/STAT3 in Kupffer cells (Stein and Yang, 1995). Furthermore, several reports have revealed the association between certain cancer progression and ER $\alpha$. Nevertheless, the targets explaining the gender disparity of HCC incidence and worsening remain elusive. In this study, we report that inhibition of G $\alpha 12$ signaling by ER $\alpha$ leads to the suppression of key aspects of both mesenchymal and amoeboid migration in HCC cells, resulting in substantial hinderance on cell motility.

G $\alpha 12$ is known to induce EMT of HCC (Yang et al., 2015), regulating pathways associated with the enhancement of pro-metastatic genes (Heo et al., 2014). In the present study, we demonstrated that ER $\alpha$ repressed the expression of $\mathrm{G} \alpha 12$. Moreover, higher $\mathrm{ER} \alpha$ and lower $\mathrm{G} \alpha 12$ levels account for the longer survival and lower recurrence rate. Consistently, human HCC samples have shown lower levels of ER $\alpha$ in HCC than in NT (Yang et al., 2015).

Deregulation of miRNAs is involved in cancer malignancy, particularly liver tumor EMT (Zaravinos, 2015). In our findings, miR-141 and -200a belonged to miRNAs exclusively regulated by both ER $\alpha$ and G $\alpha 12$ during HCC progression. miR-141 and -200a are known to regulate cancer growth and metastasis (Huang et al., 2017; Pichler et al., 2014; Li et al., 2019). Importantly, HCC cells have demonstrated increased levels of miR-141 and -200a, presenting higher expression levels in females than in males. Other molecules targeted by miR-141 and -200a are clustered into several pathways such as actin nucleation and chromosome organizations, irrelevant in our study (Supplementary 4B ).

PTP4A1 plays a role in cell proliferation and migration, cell cycle progression, and cell motility and invasion (Li et al., 2014). PTP4A1 has two other isoforms, PTP4A2, and PTP4A3, which have similar functions (Liu et al., 2016). Oncogenic PTP4A1 contains a phosphatase domain which is essential for tumor cell 
metastasis and affects focal adhesion regulation (Rios et al., 2013). PTP4A1 regulates miR-601 in breast cancer metastasis (Bai et al., 2011). In this context, the regulation of EMT remains pivotal. Furthermore, it functions in the TGF- $\beta$ pathway, contributing to fibrosis and systemic sclerosis (Hu et al., 2016). The results shown in this study support the notion that G $\alpha 12$ modulates PTP4A1 and is affected by ER $\alpha$. Therefore, through ER $\alpha$ and G $\alpha 12$, EMT phenotype is inhibited. An improved understanding of the functions and regulatory mechanism of PTP4A1 may lead to new considerations of this protein as a biomarker or therapeutic target for HCC.

In the current study, another interesting finding involves the identification of PTP4A1 as a target of miR-141 and -200a. Indeed, PTP4A1 appeared to be a specific target of miR-141 and -200a, supporting the hypothesis that the miRNAs contribute to the suppression of HCC progression through PTP4A1. Consistently, the outcomes of our cell-based assays support the idea that the miRNAs were upregulated by ER $\alpha$ ligand treatment through G $\alpha 12$ repression, but were downregulated by G $\alpha 12$ overexpression. Although the miRNAs share identical seed sequences, miR-200a is more known to play a role in cell motility (Wang et al., 2014). Interestingly, PTP4A1 seems to respond more strongly to miR-200a than miR-141. We corroborated the effects of PTP4A1 modulations on EMT. Additionally, there are other miRNAs down-regulated by G $\alpha 12$ in HCC. Among them, 15 miRNAs are known to be downregulated in HCC, 4 are related to HCC apoptosis, 6 are related to angiogenesis, and 11 are related to invasion, EMT, and HCC metastasis (Xu et al., 2018). It remains to be investigated whether the targets are regulated by $\mathrm{G} \alpha 12$.

Cancer cells can take another mode of motility, characterized as amoeboid movement (Sahai and Marshall, 2003). Amoeboid movement promotes tumor progression and is used to reprogram the immune microenvironment, another important factor in cancer metastasis (Vaškovičová et al., 2015; Georgouli et al., 2019). According to Lehmann et al., cells undergoing amoeboid movement carry EMT signatures for dissemination from the epithelial tumor. The amoeboid profile alone is insufficient to present metastasis as it is difficult to distinguish mesenchymal and amoeboid movements (Garner and de Visser, 2020). There is growing evidence focusing on the dual targeting of mesenchymal and amoeboid movements (Vaškovičová et al., 2015; Jones et al., 2017). However, limited studies have investigated amoeboid movement, especially in the field of HCC. It would be necessary to inhibit both mesenchymal and amoeboid migration and invasion for efficient therapy (Jones et al., 2017).

The gender disparity observed in HCC prognosis could be explained by differences in p-MLC, a amoeboid marker, along with alterations in PTP4A1, which may account for the metastatic capability. Indeed, the SK-Hep1 cell model that we used for our invasion/migration assays showed strong invasive power (data not shown). Hence, we were unable to differentiate alterations in cell movement in the experiment modulating $\mathrm{ER} \alpha$ and $\mathrm{G} \alpha 12$, to observe the effect of invasion and migration assays (Sacchetti et al., 2017). However, our results support the possibility that G $\alpha 12$ under the control of ER $\alpha$ modulates the p-MLC level, which may explain gender disparity in HCC exacerbation; this observation was supported by outcomes of the amoeboid cell movement video assay (Supplementary Movie 1-4).

Among molecules associated with amoeboid movement, NOX4 inhibits the transition of HCC cells to amoeboid cells and suppresses tumor dissemination (Crosas-Molist et al., 2017). CD147 promotes Src-dependent activation of Rac1 signaling during the motility of HCC (Lehmann et al., 2017). It has been proposed that simultaneous inhibition of ROCK and NEDD9 additionally decreased breast cancer cell migration and invasion (Jones et al., 2017). ROCK is one of the main downstream molecules of the Ga12 pathway and is known to facilitate a switch to amoeboid motility (Seasholtz et al., 1999; Matsuoka and Yashiro, 2014). The results showing inhibition of amoeboid cell migration and invasion by ER $\alpha$ through the Ga12 pathway, suggest an efficient approach clarifying HCC progression.

To elucidate the pathogenesis of HCC and investigate the effects of potential targets, mouse models, especially subcutaneous xenograft models, have been proposed as a useful tool (He et al., 2015). Previously, we have used a subcutaneous xenograft model to demonstrate that G $\alpha 12$ knockdown diminishes the overall growth rate (Yang et al., 2015). Here, we performed an in-depth effort to implement SK-Hep1 Tet O-ER $\alpha$ cells into SCID mice, despite which, tumor formation failed. The specific error was reportedly the attempt to 
establish solid tumors $\left(100 \mathrm{~cm}^{3}\right)$ after implanting SK-Hep1 Tet O-ER $\alpha$ cells using Charles River Balb/c nude mouse and JoongAh Bio NRGA mouse. However, HCC mouse models often experience limitations such as interference of cell growth by varied size and heterogeneity of constructs (Bi et al., 2019). Nonetheless, our analysis of the public dataset derived from a xenograft mouse model supports in vivo validity of the proposed pathway (Fig. 7A ).

Estrogen replacement therapy (ERT) is a treatment that replaces the role of hormones with medication therapy (Collaborative Group on Hormonal Factors in Breast Cancer, 2019). ERT is important to prevent later health conditions (Biglia et al., 2017). ERT may pose risks including an increase in breast cancer. However, the incidence of colorectal cancer decreases (Rossouw et al., 2002). Our findings delineate the molecular pathway of $\mathrm{ER} \alpha$ regulation of G $\alpha 12$ through the miR141/200a network. Moreover, the identified molecules displayed gender differences, suggestive of the role of ER $\alpha$ in explaining significantly low mortality in females and possible applications of estrogen. Moreover, our findings support the significance of ER $\alpha$ and $\mathrm{G} \alpha 12$ as independent prognostic factors for tumor recurrence so that the molecules and signaling axis are beneficial in understanding sex differences in HCC incidence and malignant progression. In addition, the resultant changes in amoeboid plasticity of cancer cells may explain HCC aggravation in males, and consequent better survival rates observed in females, supporting the idea that modulating the ER $\alpha$-G $\alpha 12$ pathway may be utilized for sex differential approaches in HCC treatment.

\section{References}

1. Bai, Y., Luo, Y., Liu, S., Zhang, L., Shen, K., Dong, Y., .. Zhang, Z. Y. (2011). PRL-1 protein promotes ERK1/2 and RhoA protein activation through a non-canonical interaction with the Src homology 3 domain of p115 Rho GTPase-activating protein. The Journal of Biological Chemistry , 286, 42316-42324.

2. Barreca T, Picciotto A, Franceschini R, Varagona G, Garibaldi A, Valle F, .. Rolandi E. (1993). Sex hormones and sex hormone-binding globulin in males with chronic viral hepatitis during recombinant interferon-alpha $2 \mathrm{~b}$ therapy. J Interferon Res, 13, 209- 211.

3. Bartel D. P. (2004). MicroRNAs: genomics, biogenesis, mechanism, and function. Cell , 116, 281-297.

4. Bi, Y., Shi, J., Li, S., Wang, Q., Wang, Q., Wen, X., .. Feng, H. (2019). A novel xenograft model of human HCC in immunocompetent mouse.BioRxiv, http://doi: https://doi.org/10.1101/762351.

5. Biglia N, Cagnacci A, Gambacciani M, Lello S, Maffei S, Nappi RE. (2017). Vasomotor symptoms in menopause: a biomarker of cardiovascular disease risk and other chronic diseases. Climacteric, 20, 306-312.

6. Bigsby RM. and Caperell-Grant A. (2011). The role for estrogen receptor- $\alpha$ and prolactin receptor in sex-dependent DEN-induced liver tumorigenesis. Carcinogenesis , 32, 1162- 1166.

7. Bray, F., Ferlay, J., Soerjomataram, I., Siegel, R.L., Torre, L.A. and Jemal, A. (2018). Global cancer statistics 2018: GLOBOCAN estimates of incidence and mortality worldwide for 36 cancers in 185 countries.A Cancer Journal for Clinicians , 68, 394-424.

8. Cancer Genome Atlas Research Network (2017). Comprehensive and Integrative Genomic Characterization of Hepatocellular Carcinoma. Cell, 169, 1327-1341.

9. Choi, S. S. and Diehl, A. M. (2009). Epithelial-to-mesenchymal transitions in the liver. Hepatology, 50, 2007-2013.

10. Cohen, A. and Smith, Y. (2014). Estrogen regulation of microRNAs, target genes, and microRNA expression associated with vitellogenesis in the zebrafish. Zebrafish, 11, 462-478.

11. Collaborative Group on Hormonal Factors in Breast Cancer (2019). Type and timing of menopausal hormone therapy and breast cancer risk: individual participant meta-analysis of the worldwide epidemiological evidence. Lancet, 394, 1159-1168.

12. Cooke, P. S., Nanjappa, M. K., Ko, C., Prins, G. S., and Hess, R. A. (2017). Estrogens in Male Physiology. Physiological Reviews, 97, 995-1043.

13. Crosas-Molist, E., Bertran, E., Rodriguez-Hernandez, I., Herraiz, C., Cantelli, G., Fabra, À., ... Fabregat, I. (2017). The NADPH oxidase NOX4 represses epithelial to amoeboid transition and efficient tumour dissemination. Oncogene , 36, 3002-3014. 
14. Fitzmaurice, C., Allen, C., Barber, R. M., Barregard, L., Bhutta, Z. A., Brenner, H., .. Mohsen, N. (2017). Global, regional, and national cancer incidence, mortality, years of life lost, years lived with disability, and disability-adjusted life-years for 32 cancer groups, 1990 to 2015: A systematic analysis for the global burden of disease study. JAMA Oncology, 3, 524-548.

15. Garner, H. and de Visser, K. E. (2020). Immune crosstalk in cancer progression and metastatic spread: a complex conversation. Nature Reviews. Immunology, 10.1038/s41577-019-0271-z.

16. Georgouli, M., Herraiz, C., Crosas-Molist, E., Fanshawe, B., Maiques, O., Perdrix, A., ... Sanz-Moreno, V. (2019). Regional Activation of Myosin II in Cancer Cells Drives Tumor Progression via a Secretory Cross-Talk with the Immune Microenvironment. Cell, 176, 757-774.e23.

17. Hart, M. J., Jiang, X., Kozasa, T., Roscoe, W., Singer, W. D., Gilman, A. G., .. Bollag, G. (1998). Direct stimulation of the guanine nucleotide exchange activity of p115 RhoGEF by Galpha13.Science , 280, 2112-2114.

18. He, L., Tian, D. A., Li, P. Y., and He, X. X. (2015). Mouse models of liver cancer: Progress and recommendations. Oncotarget, 6, 23306.

19. Heo, M. J., Kim, Y. M., Koo, J. H., Yang, Y. M., An, J., Lee, S. K., .. Kim, S. G. (2014). microRNA148a dysregulation discriminates poor prognosis of hepatocellular carcinoma in association with USP4 overexpression. Oncotarget, 5, 2792-2806.

20. Hu, J. Y., Yi, W., Wei, X., Zhang, M. Y., Xu, R., Zeng, L. S., .. Chen, J. S. (2016). miR-601 is a prognostic marker and suppresses cell growth and invasion by targeting PTP4A1 in breast cancer. Biomedicine 83 Pharmacotherapy, 79, 247-253.

21. Huang, F. Y., Wong, D. K., Seto, W. K., Lai, C. L., and Yuen, M. F. (2015). Estradiol induces apoptosis via activation of miRNA-23a and p53: implication for gender difference in liver cancer development. Oncotarget, 6, 34941-34952.

22. Huang, S., Wa, Q., Pan, J., Peng, X., Ren, D., Huang, Y., ... Tang, Y. (2017). Downregulation of miR-141-3p promotes bone metastasis via activating NF- $\varkappa \mathrm{B}$ signaling in prostate cancer. Journal of Experimental \& Clinical Cancer Research, 36, 173.

23. Jones, B. C., Kelley, L. C., Loskutov, Y. V., Marinak, K. M., Kozyreva, V. K., Smolkin, M. B., and Pugacheva, E. N. (2017). Dual Targeting of Mesenchymal and Amoeboid Motility Hinders Metastatic Behavior. Molecular Cancer Research, 15, 670-682.

24. Kehl, T., Backes, C., Kern, F., Fehlmann, T., Ludwig, N., Meese, E., ... Keller, A. (2017). About miRNAs, miRNA seeds, target genes and target pathways. Oncotarget, 8, 107167-107175.

25. Lehmann, S., Te Boekhorst, V., Odenthal, J., Bianchi, R., van Helvert, S., Ikenberg, K., .. F Friedl, P. (2017). Hypoxia Induces a HIF-1-Dependent Transition from Collective-to-Amoeboid Dissemination in Epithelial Cancer Cells. Current Biology , 27, 392-400.

26. Li, H., Tang, J., Lei, H., Cai, P., Zhu, H., Li, B., .. Tang, W. (2014). Decreased MiR-200a/141 suppress cell migration and proliferation by targeting PTEN in Hirschsprung's disease. Cellular Physiology and Biochemistry , 34, 543-553.

27. Li, W., Cui, Y., Wang, D., Wang, Y., and Wang, L. (2019). MiR-141-3p functions as a tumor suppressor through directly targeting ZFR in non-small cell lung cancer. Biochemical and Biophysical Research Communications, 509, 647-656.

28. Liu, L. Z., He, Y. Z., Dong, P. P., Ma, L. J., Wang, Z. C., Liu, X. Y., .. Wang, X-Y. (2016). Protein tyrosine phosphatase PTP4A1 promotes proliferation and epithelial-mesenchymal transition in intrahepatic cholangiocarcinoma via the PI3K/AKT pathway. Oncotarget, 7, 75210-75220.

29. Liu, W. H., Yeh, S. H., Lu, C. C., Yu, S. L., Chen, H. Y., Lin, C. Y., .. Chen, P. J. (2009). MicroRNA-18a prevents estrogen receptor-alpha expression, promoting proliferation of hepatocellular carcinoma cells. Gastroenterology , 136, 683-693.

30. Liu, Y. J., Le Berre, M., Lautenschlaeger, F., Maiuri, P., Callan-Jones, A., Heuzé, M., ... Piel, M. (2015). Confinement and low adhesion induce fast amoeboid migration of slow mesenchymal cells. Cell, $160,659-672$.

31. Matsuoka T. and Yashiro M. (2014). Rho/ROCK signaling in motility and metastasis of gastric cancer. World J Gastroenterol, 20, 13756-13766. 
32. Natri, H. M., Wilson, M. A., and Buetow, K. H. (2019). Distinct molecular etiologies of male and female hepatocellular carcinoma.BMC Cancer, 19, 951.

33. Naugler, W. E., Sakurai, T., Kim, S., Maeda, S., Kim, K., Elsharkawy, A. M., and Karin, M. (2007). Gender disparity in liver cancer due to sex differences in MyD88-dependent IL-6 production. Science,317, 121-124.

34. Nieto MA. (2013). Epithelial plasticity: a common theme in embryonic and cancer cells. Science, 342, 1234850.

35. Nobes, C. D., and Hall, A. (1995). Rho, rac, and cdc42 GTPases regulate the assembly of multimolecular focal complexes associated with actin stress fibers, lamellipodia, and filopodia. Cell,81, 53-62.

36. Pankova, K., Rosel, D., Novotny, M., and Brabek, J. (2010). The molecular mechanisms of transition between mesenchymal and amoeboid invasiveness in tumor cells. Cellular and Molecular Life Sciences, $67,63-71$.

37. Pichler, M., Ress, A. L., Winter, E., Stiegelbauer, V., Karbiener, M., Schwarzenbacher, D., ... Hoefler, G. (2014). MiR-200a regulates epithelial to mesenchymal transition-related gene expression and determines prognosis in colorectal cancer patients. British Journal of Cancer, 110, 1614-1621.

38. Poincloux, R., Collin, O., Lizarraga, F., Romao, M., Debray, M., Piel, M., and Chavrier, P. (2011). Contractility of the cell rear drives invasion of breast tumor cells in 3D Matrigel. Proceedings of the National Academy of Sciences of the United States of America , 108(5), 1943-1948.

39. Rios, P., Li, X., and Kohn, M. (2013). Molecular mechanisms of the PRL phosphatases. The FEBS Journal , 280(2), 505-524.

40. Rossouw JE, Anderson GL, Prentice RL, LaCroix AZ, Kooperberg C, Stefanick ML, ... Writing Group for the Women's Health Initiative Investigators. (2002). Risks and benefits of estrogen plus progestin in healthy postmenopausal women: principal results from the Women's Health Initiative randomized controlled trial. JAMA, 288, 321-333.

41. Sacchetti, C., Bai, Y., Stanford, S. M., Di Benedetto, P., Cipriani, P., Santelli, E., .. Bottini, N. (2017). PTP4A1 promotes TGF $\beta$ signaling and fibrosis in systemic sclerosis. Nature Communications, $8(1), 1060$.

42. Sahai, E., and Marshall, C. J. (2003). Differing modes of tumour cell invasion have distinct requirements for Rho/ROCK signalling and extracellular proteolysis. Nature Cell Biology, 5(8), 711-719.

43. Seasholtz, T. M., Majumdar, M., and Brown, J. H. (1999). Rho as a mediator of G protein-coupled receptor signaling. Molecular Pharmacology, 55(6), 949-956.

44. Song, D. G., Lee, G. H., Nam, S. H., Cheong, J. G., Jeong, D., Lee, S. J., .. Lee, J.W. (2017). TM4SF5 promotes metastatic behavior of cells in 3D extracellular matrix gels by reducing dependency on environmental cues. Oncotarget, 8(48), 83480-83494.

45. Stein, B. and Yang, M. X. (1995). Repression of the interleukin-6 promoter by estrogen receptor is mediated by NF-kappa B and C/EBP beta. Molecular and Cellular Biology, 15(9), 4971-4979.

46. Thiery J, Acloque H, Huang R, and Nieto M. (2009). Epithelial-mesenchymal transitions in development and disease. Cell, 139(5), 871-90.

47. Vaškovičová, K., Szabadosová, E., Čermák, V., Gandalovičová, A., Kasalová, L., Rösel, D., and Brábek, J. (2015). PKC $\alpha$ promotes the mesenchymal to amoeboid transition and increases cancer cell invasiveness. BMC Cancer, $15,326$.

48. Wang S., Cui H., Liu Y., Zhao P., Zhang Y., Fu Z., . . Jiang J. (2014). CD147 promotes Src-dependent activation of Rac1 signaling through STAT3/DOCK8 during the motility of hepatocellular carcinoma cells. Oncotarget, 6, 243-257.

49. Wilkinson, S., Paterson, H. F., and Marshall, C. J. (2005). Cdc42-MRCK and Rho-ROCK signalling cooperate in myosin phosphorylation and cell invasion. Nature Cell Biology, 7(3), 255-261.

50. Wu, H., Yao, S., Zhang, S., Wang, J. R., Guo, P. D., Li, X. M., .. Li, J. M. (2017). Elevated expression of Erbin destabilizes ER $\alpha$ protein and promotes tumorigenesis in hepatocellular carcinoma. Journal of Hepatology, 66(6), 1193-1204.

51. Xu, X., Tao, Y., Shan, L., Chen, R., Jiang, H., Qian, Z., .. Yu, Y. (2018). The Role of MicroRNAs in Hepatocellular Carcinoma.Journal of Cancer, 9(19), 3557-3569. 
52. Yang, B., Li, M., Tang, W., Liu, W., Zhang, S., Chen, L., and Xia, J. (2018). Dynamic network biomarker indicates pulmonary metastasis at the tipping point of hepatocellular carcinoma. Nature Communications, 9(1), 678.

53. Yang, W., Lu, Y., Xu, Y., Xu, L., Zheng, W., Wu, Y., ... Shen, P. (2012). Estrogen represses hepatocellular carcinoma ( $\mathrm{HCC}$ ) growth via inhibiting alternative activation of tumor-associated macrophages (TAMs). Journal of Biological Chemistry, 287(48), 40140-40149.

54. Yang, Y. M., Lee, W. H., Lee, C. G., An, J., Kim, E. S., Kim, S. H., .. Kim, S. G. (2015). G $\alpha 12$ gep oncogene deregulation of p53-responsive microRNAs promotes epithelial-mesenchymal transition of hepatocellular carcinoma. Oncogene , 34(22), 2910-2921.

55. Zaravinos A. (2015). The Regulatory Role of MicroRNAs in EMT and Cancer. Journal of Oncology, 2015,865816 .

Figure legends

Figure 1. Inverse correlation between $\mathrm{ER} \alpha$ and $\mathrm{G} \alpha 12$ expression in $\mathrm{HCC}$

(A) Heat map showing significantly increased and decreased mRNAs across the progression of HCC using the GEO database (GSE40367; Roessler S.et al. , 2012) and KEGG pathway: pathways in cancer. Colors represent $\log 2$ gene expression fold change in normal controls when compared with non-metastatic HCC and metastatic HCC patients. A $\log 2$ fold change of more than 2 or less than -2 was considered overexpression or suppression (blue, underexpression; white, no change; and red, overexpression; $\mathrm{n}=5$ for normal, $\mathrm{n}=10$ for non-metastatic $\mathrm{HCC}$, and $\mathrm{n}=20$ for metastatic $\mathrm{HCC}$ ).

(B) An interaction map of ESR1 and highly changed genes from HCC progression. A gene interaction network of ESR1 and genes from panel (A), revealing the unidentified interaction between GNA12and ESR1 using a biological database STRING. The interaction map is drawn using Cytoscape. Each line indicates known and predicted association.

(C) GSEA-enrichment plots showing enriched signaling pathways in males (versus females) HCC patients (GSE76427; Grinchuk OV, et al. , 2018). Gene sets involved in EMT hallmark (left ) and GPCR ligand binding receptor were positively correlated with HCC males (right, $\mathrm{n}=21$ females, and 94 males) (left, $\mathrm{NES}=1.61, \mathrm{FDR}<0.25 ;$ right $, \mathrm{NES}=2.28, \mathrm{FDR}<0.25)$.

(D) Pearson correlation analyses showing negative relationship between GNA12 and estrogen receptor isoforms. (Upper ) Using human HCC GEO dataset from Fig. 1A, correlations between estrogen receptorsESR1 , ESR2, and GPER1 and GNA12 were investigated. Correlations were analyzed by Pearson correlation analysis (r). Each point represents one sample, and the horizontal line indicates the mean value $(\mathrm{n}=35)$. (Lower ) Moreover, screened for relationships between estrogen receptors (ESR1 , ESR2, andGPER1) in HCC patients with or without metastasis (or normal controls) deposited in GEO database (GSE40367, Roessler S. et al. , 2012) ( $\mathrm{n}=5$ for normal, $\mathrm{n}=10$ for non-metastatic HCC, and $\mathrm{n}=20$ for metastatic HCC). The horizontal line indicates the mean value. Data were analyzed using the ANOVA.

(E) Relationship between GNA12 and ESR1 in patients with HCC using the GEO dataset (GSE54236, Villa E. et al. , 2016). The samples were divided into two groups of high and low gene expression according to qRT-PCR assay results for GNA12 and ESR1. The cutoff for high low expression was defined by the median. The data were analyzed using the Chi-square test.

Figure 2. Survival rates of patients with $\mathrm{HCC}$ in association with $\mathrm{ER} \alpha$ and $\mathrm{G} \alpha 12$ expression

(A) Kaplan-Meier analyses of HCC patients. The overall survival curves and recurrence free survival rates of HCC patients from Asan Medical Center (left , $\mathrm{n}=59$ ) and in HCC GEO database (right, GSE76427, Grinchuk OV, et al. , 2018) in terms of G $\alpha 12$ and ER $\alpha$ expression ( $\mathrm{n}=21$ females, and 94 males). HCC patients are grouped by high $\mathrm{ER} \alpha$ and low $\mathrm{G} \alpha 12$, or low $\mathrm{ER} \alpha$ and high $\mathrm{G} \alpha 12$ expression. The cutoff for strong Ga12 intensity was set at a ' $>3$-fold' difference in human tumourous HCC (T) / non-tumorous $(\mathrm{N})_{\text {avg. }}$ P-value was generated by the log-rank test. 
(B) Pearson correlation analyses to explore relationship amongESR1 and EMT related genes, GNA12 and VIM . HCC patients. ESR1 and VIM (left), and VIM and GNA12 using GEO database (GSE40367, Roessler S.et al. , 2012) (right, $\mathrm{n}=35$ ). Correlations were analyzed by using pearson correlation analysis (r). Each point represents one sample.

(C) Immunoblottings for $\mathrm{G} \alpha 12$ and $\mathrm{ER} \alpha$ in $\mathrm{T}$ and $\mathrm{N}$ samples. Protein levels were measured in 59 pairs of primary Ts and Ns. ${ }^{*} \mathrm{p}<0.05$ and ${ }^{* * *} \mathrm{p}<0.001$. Data were analyzed using the two-tailed Student's t-test.

(D) IHC for $\mathrm{ER} \alpha$ and $\mathrm{G} \alpha 12$ (x 200). ER $\alpha$ and $\mathrm{G} \alpha 12$ protein levels were analyzed by IHC in T tissues (versus $\mathrm{N}$ tissues). ( $\mathrm{n}=3,4-5$ replicates/group for each experiment, Scale bar, $200 \mu \mathrm{m})$. Pictures are representative images. ${ }^{*} \mathrm{p}<0.05$ and ${ }^{* *} \mathrm{p}<0.01$. Data were analyzed using the two-tailed Student's t-test.

Figure 3. Transcriptional inhibition of $\mathrm{G} \alpha 12$ by ER $\alpha$

(A) Schematic drawing illustrating the principle of the Tet-On system used to ectopically express ER $\alpha$ in SK-Hep1 cells (left ). qRT-PCR assays for GNA12 in SK-Hep1 Tet-on system for ER $\alpha$ (Tet O-Er $\alpha$ ) cells treated with $2 \mathrm{mg} / \mu \mathrm{l}$ Dox (or Veh) and continuously treated with $100 \mathrm{nM}$ E2 for $24 \mathrm{~h}$ each (right, $\mathrm{n}=3$ each, $\left.{ }^{*} \mathrm{p}<0.05\right)$. Data were analyzed using the two-tailed Student's t-test.

(B) qRT-PCR assays for GNA12 in SNU449 and HepG2 cells transfected with control and ER $\alpha$ O/E vector $1 \mu \mathrm{g}$ for $24 \mathrm{~h}$ (left), and si ER $\alpha$ plasmid, $1 \mu \mathrm{g}$ for $72 \mathrm{~h}$ (right) and 18S rRNA was used as internal control $\left(\mathrm{n}=3\right.$ each, ${ }^{*} \mathrm{p}<0.05$ and $\left.{ }^{* *} \mathrm{p}<0.01\right)$. Data were analyzed using the two-tailed Student's t-test.

(C) qRT-PCR assays for GNA12 in SNU 387 cells treated with $100 \mathrm{nM}$ E2 in a time-dependent manner for $24 \mathrm{~h}\left(\mathrm{n}=3\right.$ each). 18S rRNA was used as internal control $\left({ }^{*} \mathrm{p}<0.05\right.$ and $\left.{ }^{* * *} \mathrm{p}<0.001\right)$. Data were analyzed using the ANOVA.

(D) ERE binding to GNA12 promoter. ChIP assays were done on the lysates of SK-Hep1 Tet O-ER $\alpha$ cells treated with $2 \mathrm{mg} / \mu \mathrm{l}$ Dox for $24 \mathrm{~h}$ and $100 \mathrm{nM}$ E2 for $24 \mathrm{~h}$ (left). DNA-protein complexes were precipitated with anti-ER $\alpha$ antibody and subjected to PCR amplification using the flanking primers for the ERE binding. IgG immunoprecipitation represents negative control and their quantifications (right) $\left(\mathrm{n}=3\right.$ each, ${ }^{*} \mathrm{p}<0.05$ and $\left.{ }^{* * *} \mathrm{p}<0.001\right)$. Data were analyzed using the two-tailed Student's t-test.

(E) Luciferase assay structure is shown on the top and the relative luciferase activity measured on the bottom ( $\mathrm{n}=3$ each, $\left.{ }^{* *} \mathrm{p}<0.01\right)$. Data were analyzed using the two-tailed Student's t-test.

(F) Confocal images of Ga12 (green) and $\mathrm{ER} \alpha$ (red), and Hoechst 33342 (blue) immunostaining in SKHep1 Tet O-ER $\alpha$ cells with $2 \mathrm{mg} / \mu \mathrm{l}$ Dox (or Veh) and $100 \mathrm{nM}$ E2 treated for $24 \mathrm{~h}$ each (n =3 each, 4-5 replicates/group for each experiment; ${ }^{*} \mathrm{p}<0.05$ and $\left.{ }^{* * *} \mathrm{p}<0.001\right)$. Data were analyzed using the two-tailed Student's t-test.

Figure 4. ER $\alpha$ regulation of EMT biomarkers via G $\alpha 12$

(A) Immunoblottings for G $\alpha 12$ and EMT markers using SK-Hep1 Tet O-ER $\alpha$ cells, treated with $2 \mathrm{mg} / \mu \mathrm{l}$ Dox and continuously treated with $100 \mathrm{nM}$ E2 for nuclearization of ER $\alpha$ for $24 \mathrm{~h}$ each, as well as their quantifications (right) $\left(\mathrm{n}=3\right.$ each, $\left.{ }^{*} \mathrm{p}<0.05\right)$. Data were analyzed using the two-tailed Student's t-test.

(B) Immunoblottings for G $\alpha 12$ and EMT markers performed using SK-Hep1 Tet O-ER $\alpha$ cells transfected with Ga12 O/E plasmid (1 $\mu \mathrm{g})$ or pCMV (Mock) $(1 \mu \mathrm{g})$, treated with $2 \mathrm{mg} / \mu \mathrm{l}$ Dox and continuously treated with $100 \mathrm{nM} \mathrm{E} 2$ for nuclearization of $\mathrm{ER} \alpha$ for $24 \mathrm{~h}$ each, as well as their quantifications (right ) ( $\mathrm{n}=3$ each, $* \mathrm{p}<0.05)$. Data were analyzed using the ANOVA.

(C) Immunoblottings for Ga12 and EMT markers performed using SNU449 cells transiently transfected with ER $\alpha$ or pcDNA (WT) (left ), or SNU449 cells stably transfected with ER $\alpha$ O/E plasmid (1 $\mu \mathrm{g}$ ) or Mock (right) for $24 \mathrm{~h}$ and continuously treated with $100 \mathrm{nM}$ E2 for nuclearization of ER $\alpha$ for $24 \mathrm{~h}$ (repeated experiments). 
(D) Immunoblottings for G $\alpha 12$ and EMT markers in HepG2 cells transfected with si ER $\alpha$ or control for 72 $\mathrm{h}$ (left) and their quantifications (right) $\left(\mathrm{n}=3\right.$ each, $\left.{ }^{*} \mathrm{p}<0.05\right)$. Data were analyzed using the two-tailed Student's t-test.

(E) Immunoblotting for G $\alpha 12$ and EMT markers in SNU387 cells (female HCC cell line) treated with 100 nM E2 for the indicated times (repeated experiments).

(F) Immunoblottings for G $\alpha 12$ and EMT markers in SNU387 cells treated with E2 with or without ICI 182780, followed by $100 \mathrm{nM}$ E2 treatment for $24 \mathrm{~h}$ and $1 \mathrm{mM}$ ICI 182780 for $1 \mathrm{~h}$ before E2 treatment (repeated experiments).

(G) Immunoblottings for ER $\alpha, \mathrm{G} \alpha 12$, and EMT markers in HCC-derived cell lines (repeated experiments).

Figure 5. miR-141 and -200a as inhibitors of PTP4A1 downregulation of ER $\alpha-\mathrm{G} \alpha 12$ axis

(A) Venn diagram of miR-141 and -200a expression among the upregulated and downregulated miRNAs induced by $100 \mathrm{nM} \mathrm{E} 2$ treatment and G $\alpha 12 \mathrm{QL}$. G $\alpha 12 \mathrm{QL}$ is an activated oncogenic mutant of G $\alpha 12$. Notably, 42 miRNAs are up-regulated in the first set of estrogen-treated SNU387 cells; 104 miRNAs are up-regulated in the second set of estrogen-treated zebrafish; 138 miRNAs are down-regulated in the Ga12QL-Huh7 cells.

(B) qRT-PCR assays for ER $\alpha$ effects on miR-141 and -200a. miR-141 and -200a miRNA levels are increased by stable ER $\alpha$ overexpression on SNU449 cells (left ). These cells were treated with $100 \mathrm{nM}$ E2 for the nuclearization of $\mathrm{ER} \alpha$ for $24 \mathrm{~h}$. Decreased levels of miR-141 and -200a miRNA levels are observed by following knockdown of ER $\alpha$ in HepG2 cells for $72 \mathrm{~h}$ (right). U6 small nuclear RNA was used as a normalizing reference for miRNA ( $\mathrm{n}=3$ each, ${ }^{*} \mathrm{p}<0.05,{ }^{* *} \mathrm{p}<0.01$, and $\left.{ }^{* * *} \mathrm{p}<0.001\right)$. Data were analyzed using the two-tailed Student's t-test.

(C) qRT-PCR assays for estrogen effect on miR-141 and -200a. Increase in miR-141 and -200a miRNA levels following treatment with $100 \mathrm{nM} \mathrm{E2}$ at times indicated using SNU387 cells $\left(\mathrm{n}=3\right.$ each, ${ }^{*} \mathrm{p}<0.05$ and $* * \mathrm{p}<0.01)$. Data were analyzed using the ANOVA.

(D) qRT-PCR assays for exploring Ga12 effects on miR-141 and -200a. SNU449 cells were transfected with control siRNA (siCON) or siRNA directed against G $\alpha 12$ (si G $\alpha 12)$. Transfection was done for $48 \mathrm{~h}(\mathrm{n}=3$ each, $\left.{ }^{*} \mathrm{p}<0.05\right)$. Data were analyzed using the two-tailed Student's t-test.

(E) qRT-PCR assays for showing miR-141 and -200a in control expression and SK-Hep1 Tet O-ER $\alpha$ cells with pCMV and Ga12 overexpression transfection for $24 \mathrm{~h}$, followed by $2 \mathrm{mg} / \mu \mathrm{l}$ Dox and $100 \mathrm{nM}$ E2 treatment for $24 \mathrm{~h}$ each $\left(\mathrm{n}=3\right.$ each, ${ }^{*} \mathrm{p}<0.05$ and $\left.{ }^{* *} \mathrm{p}<0.01\right)$. Data were analyzed using the ANOVA.

(F) An integrative network of putative or validated targets of miR-141 and -200a. Five hundred miRtargetable transcripts were analyzed using DAVID GO. From the significant pathways, biological process: regulation in cell migration was used to determine possible EMT regulators. Among the nine transcripts in the regulation in cell migration list, STRING analysis was used to distinguish the unknown yet important molecules to resolve EMT in HCC. Here, nodes represent genes/proteins, whereas edges represent interactions. Specifically, red nodes represent up-regulation in HCC; purples represent down-regulation in HCC; greens represent unknown in HCC; yellow circles represent involvement in migration; black circles represent predicted miR-141 and -200a binding targets.

(G) Prediction of miR-141 and -200a binding to the 3'-UTR of human PTP4A1 mRNA (left). The effect of miR-141 and -200a mimic and ASO treatment on PTP4A1 expression. Immunoblottings for PTP4A1 were done using SNU449 and HepG2 cells transfected with miR-141 and -200a mimic, ASO, or the respective control for $48 \mathrm{~h}$ ( right, $\mathrm{n}=3$ each, $\left.{ }^{*} \mathrm{p}<0.05\right)$. Data were analyzed using the ANOVA.

(H) Immunoblottings for the effect of ER $\alpha$ on PTP4A1 expression. PTP4A1 was measured on SK-Hep1 Tet O-ER $\alpha$ cells treated with $2 \mathrm{mg} / \mu \mathrm{l}$ Dox for $24 \mathrm{~h}$ and continuously treated with $100 \mathrm{nM}$ E2 for $24 \mathrm{~h}$ (left ). PTP4A1 was measured on HepG2 cells with siER $\alpha$ transfected for $72 \mathrm{~h}$ (right, repeated experiments). 
(I) Immunoblottings for the effect of G $\alpha 12$ overexpression transfection on PTP4A1 with ER $\alpha$ modulation treated with $2 \mathrm{mg} / \mu \mathrm{l}$ Dox for $24 \mathrm{~h}$ and continuously treated with $100 \mathrm{nM} \mathrm{E} 2$ for $24 \mathrm{~h}$ (repeated experiments).

(J) Immunoblottings for the effect of PTP4A1 modulation with indicated PTP4A1 O/E or siPTP4A1 on EMT markers. PTP4A1 O/E vector $(1 \mu \mathrm{g})$ was transfected on HepG2 cells for $48 \mathrm{~h}$, and PTP4A1 was silenced on SNU449 cells for $72 \mathrm{~h}$ (repeated experiments).

Figure 6. Inhibition of amoeboid movement by ER $\alpha$ through $\mathrm{G} \alpha 12$

(A) Schematic drawing of cancer cells gaining invasiveness through phenotypic changes using matrix metallopeptidases (MMPs) or amoeboid transition.

(B) GSEA of HCC GEO data (GSE9843; Chiang DY, et al. , 2008) depicting pathway signatures of MAT positively correlated with male (versus female) HCC patients ( $\mathrm{n}=27$ females, and 53 males) (left, NES $=1.91, \mathrm{FDR}<0.25$; and right, NES $=1.57$, FDR <0.25).

(C) 3-dimensional imaging of SK-Hep1 Tet O-ER $\alpha$ cells treated with $2 \mathrm{mg} / \mu \mathrm{l}$ Dox (or Veh) for $24 \mathrm{~h}$, and continuously treated with $100 \mathrm{nM} \mathrm{E} 2$ for $24 \mathrm{~h}$ in a collagen I matrix $\left(8^{\sim} 10 \mathrm{mg} / \mathrm{ml}\right)$ (left ). Prior to imaging using time-lapse microscopy with controlled $\mathrm{CO}_{2}(5 \%)$ and temperature $\left(37^{\circ} \mathrm{C}\right)$ for $24 \mathrm{~h}$ or indicated times. The representative end point images are shown. Using ImageJ software embedded program ADAPT, blebbing and its velocity were measured throughout the time-lapse imaging for $24 \mathrm{~h}$ ( right, $\mathrm{n}=3$ each, ${ }^{*} \mathrm{p}<0.05$ and $* * * \mathrm{p}<0.001)$. Data were analyzed using the two-tailed Student's t-test.

(D) Representative confocal images of p-MLC (green), G $\alpha 12$ (red) and Hoechst (blue) staining ( $\mathrm{n}=3$ each, ${ }^{*} \mathrm{p}<0.05$ and $\left.{ }^{* *} \mathrm{p}<0.01\right)$. Data were analyzed using the two-tailed Student's t-test.

(E) Immunoblottings showing p-MLC expression in ER $\alpha$ overexpression SK-Hep1 cells with Tet-On ER $\alpha$ system for elucidating the effect of $\mathrm{ER} \alpha$ on amoeboid movements (left ). Immunoblotting showing p-MLC expression in ER $\alpha$ knockdown HepG2 cells for $72 \mathrm{~h}$ (right ).

(F) Immunoblotting for the expression of p-MLC by modulating ER $\alpha$ and G $\alpha 12$. ( $\mathrm{n}=3$ each, ${ }^{*} \mathrm{p}<0.05$, $\left.{ }^{* *} \mathrm{p}<0.01,{ }^{* * *} \mathrm{p}<0.001\right)$. Data were analyzed using the ANOVA.

Figure 7. Changes of identified biomarkers under the control of ER $\alpha$ in animal dataset publicly available and human HCC samples

(A) Pearson correlation analyses for the relationships among ESR1, GNA12 and PTP4A1 in mouse xenograft GEO dataset (GSE94016, Yang B., et al. , 2018) ( $\mathrm{n}=5$ animals, 5 replicates). Gene expression was measured at five different time points as metastasis occurs. HCCLM3-REP mouse model was used and pulmonary metastasis was observed. Correlations were assessed using Pearson correlation analysis (r). Each point represents five samples at five different metastasis points.

(B) Immunoblottings for PTP4A1 and p-MLC. Protein levels were measured in 59 pairs of primary tumorous HCCs $(\mathrm{T})$ and non-tumorous $(\mathrm{N})$ specimens $\left({ }^{*} \mathrm{p}<0.05\right.$ and $\left.{ }^{* *} \mathrm{p}<0.01\right)$. Data were analyzed using the twotailed Student's t-test.

(C) IHC for PTP4A1 and p-MLC $(\times 200)(\mathrm{n}=3$ each, 4-5 replicates/group for each experiment, Scale bar, $100 \mu \mathrm{m})\left({ }^{*} \mathrm{p}<0.05\right.$ and $\left.{ }^{* *} \mathrm{p}<0.01\right)$. Data were analyzed using the two-tailed Student's t-test.

(D) qRT-PCR assays to show gender disparity in the expression of newly found miR-141 and -200a (left ). The ratio of $\mathrm{T} / \mathrm{N}$ is lowly expressed in miR-141 and -200a in male (versus female) HCC patient samples. Data were analyzed using the Pearson correlation analysis. Symbols represent individual samples. qRT-PCR assays to explore the relationship of newly found miR-141 and -200a. Significantly positive correlations are observed between ESR1 and miR-141 and -200a in the HCC samples (right, $\mathrm{n}=59,{ }^{*} \mathrm{p}<0.05$ and ${ }^{* *} \mathrm{p}<0.01$ ). Data were analyzed using the two-tailed Student's t-test.

(E) Quantification of Ga12, PTP4A1, and p-MLC protein expression by sex. Higher expression of Ga12, PTP4A1, and p-MLC is observed in male (M) (versus female (F)) HCC patients without microvascular 
invasion ( $\mathrm{n}=40,27$ males, and 13 females, $\left.{ }^{* *} \mathrm{p}<0.01\right)$. Data were analyzed using the two-tailed Student's t-test. Outliers were removed with a range $>1.5$ interquartile range.

(F) The schematic drawing the role of ER $\alpha-G \alpha 12$ in HCC progression through regulation of miR-141/200a/PTP4Al and amoeboid movement.

A)

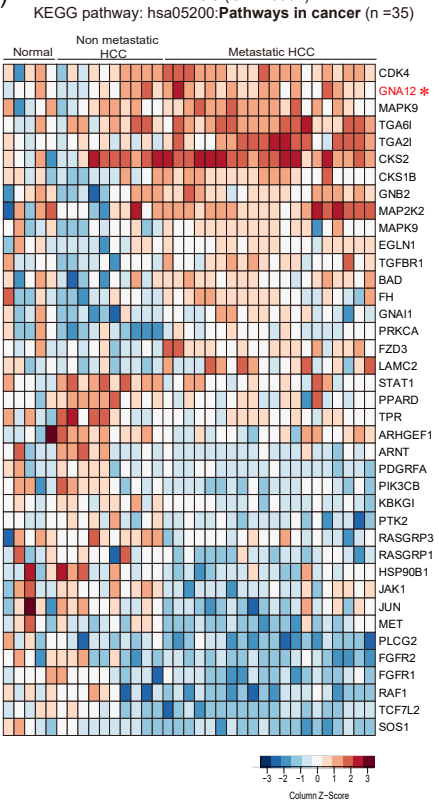

D)

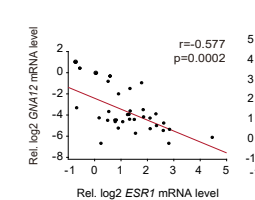

Human HCC ( $n=35$; GSE40367)

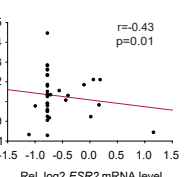

5
4
3
2
1
0
-1

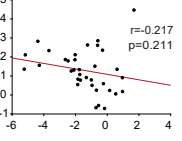

Rel. log2 GPER1 mRNA level

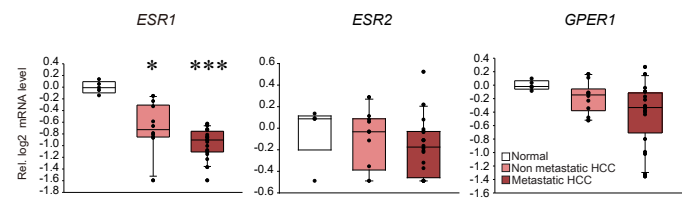

B)

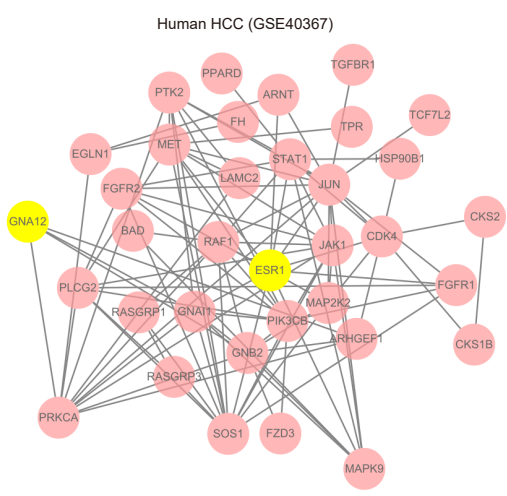

C)

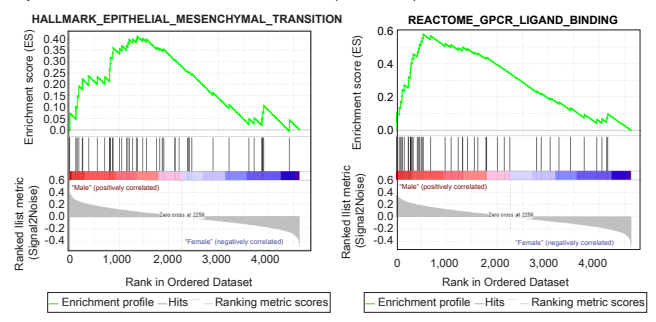

E)

Human HCC (GSE54236) $\square$ Low GNA12 expression
$\square$ High GNA12 expression

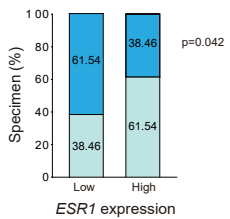

Figure 1

\section{Hosted file}

figure 2.pdf available at https://authorea.com/users/378507/articles/494981-estrogenreceptor-alpha-inhibits-mesenchymal-and-amoeboidal-movement-of-liver-cancer-cell-viag-protein-subunit-alpha-12 
A)
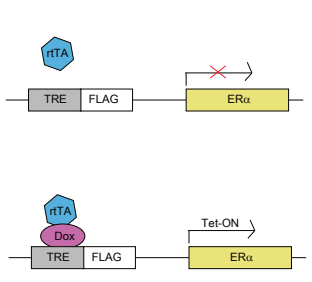

C)

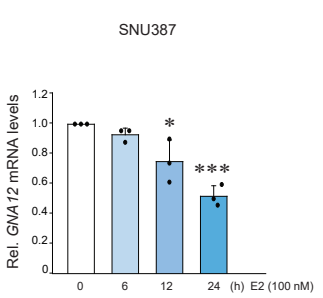

B)

SK-Hep1 Tet O-ER $\alpha$
E2 $(100 \mathrm{nM})$

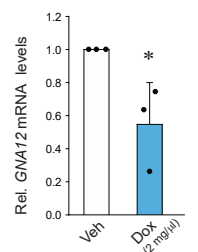

SNU449

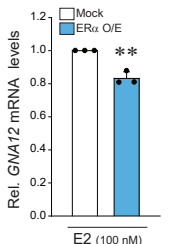

HepG2

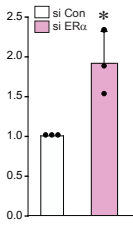

D)
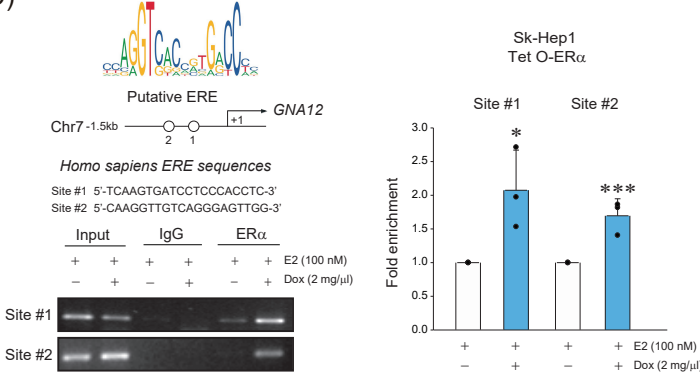

E)

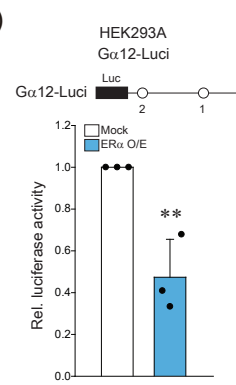

F)
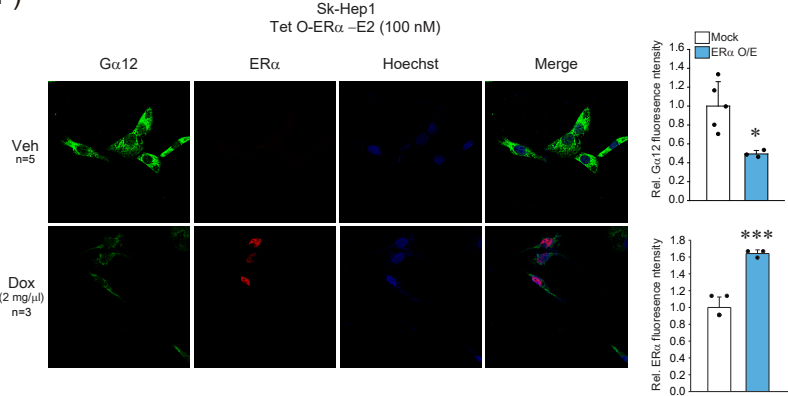

Figure 3 
A)

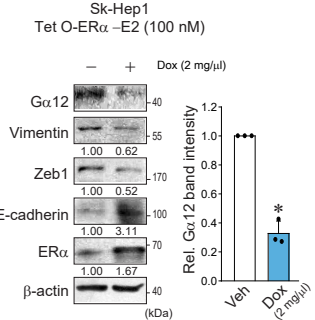

C)

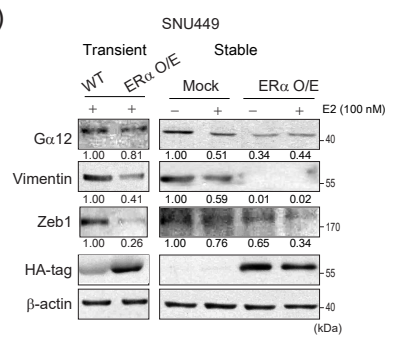

B)

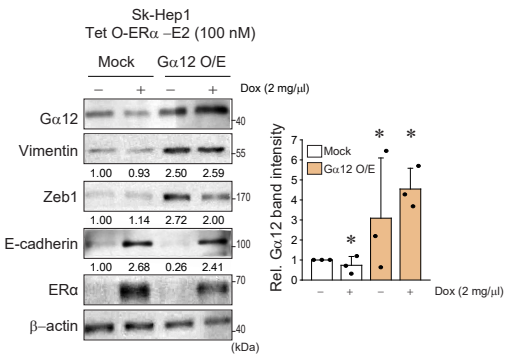

D)

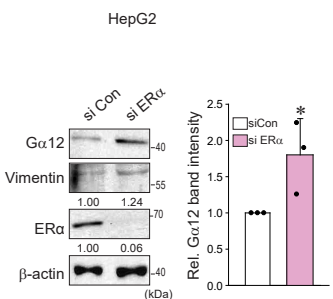

G)

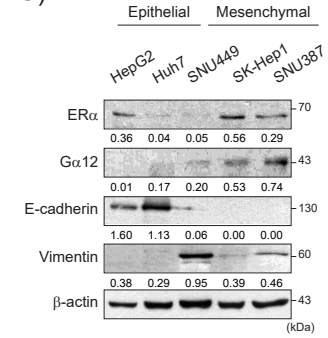

Figure 4 
A)

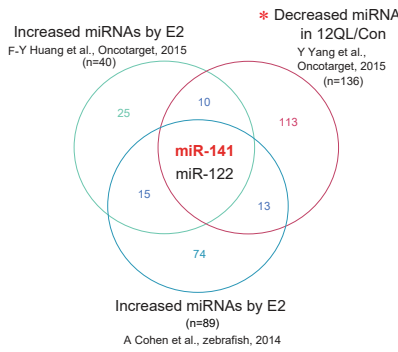

C)
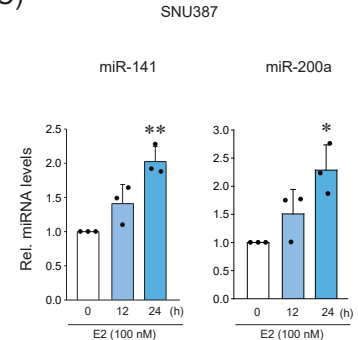

F) GO: Regulation of cell migration

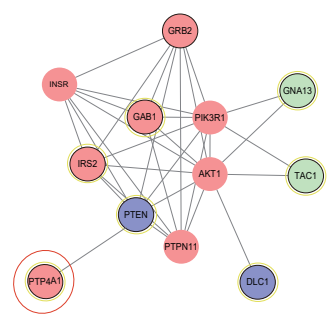

H)

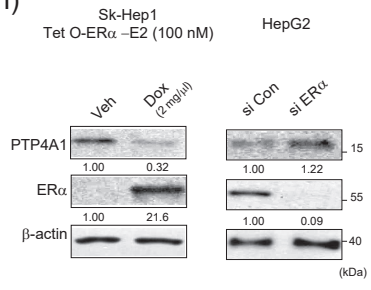

D)

G)

I)
B)
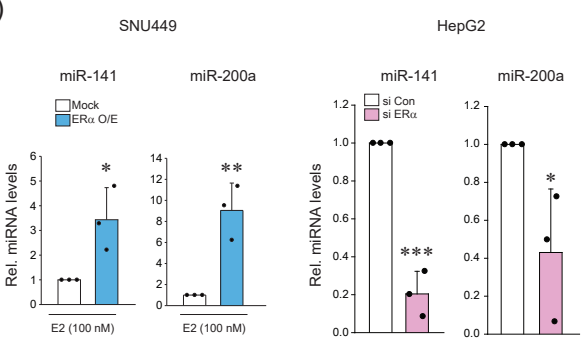

SNU449

E)

miR-141 miR-200a

Sk-Hep1 Tet O-ERa E2 (100 nM)

miR-141 miR-200a

$\square_{\square}^{\text {Mock }} \alpha 12$ O/E
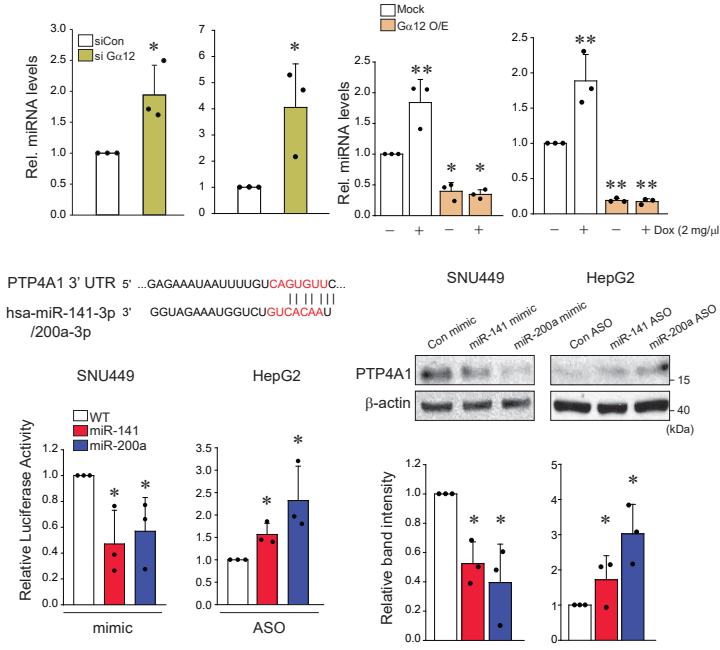

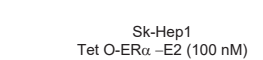

J)

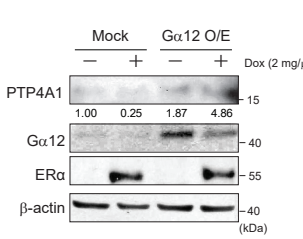

Figure 5 
A)

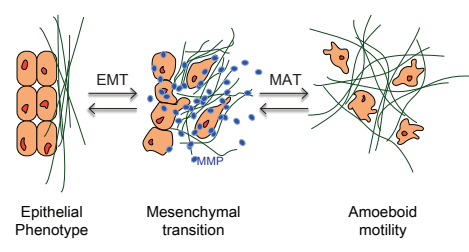

C)

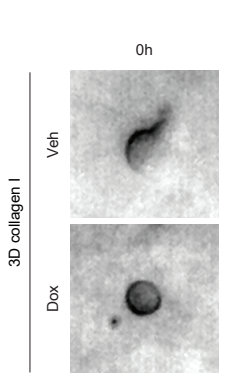

D)

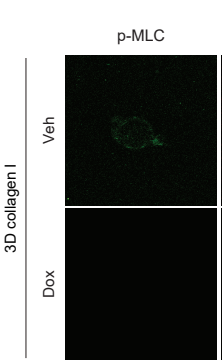

E)

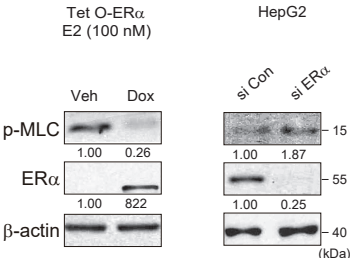

B)

Human HCC (GSE9843)

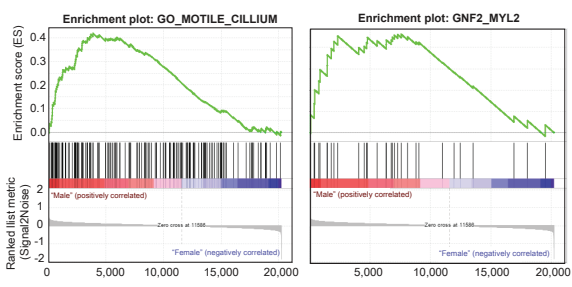

\begin{tabular}{|l|l}
\hline Enrichment profile & Hits \\
\hline
\end{tabular}

Tet O-ER $\alpha$ E2 (100 nM)
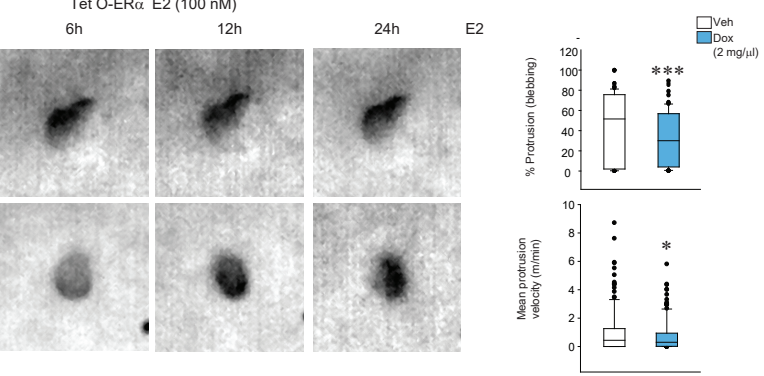

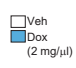

